\title{
Jupiter's Temperate Belt/Zone Contrasts Revealed at Depth by Juno Microwave Observations
}

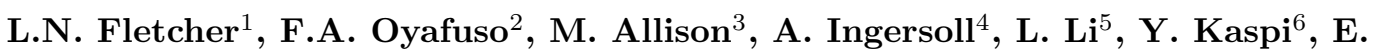

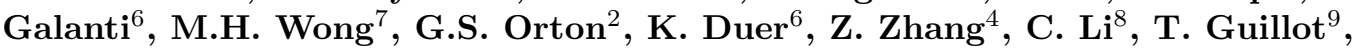
S.M. Levin ${ }^{2}$, S. Bolton ${ }^{10}$

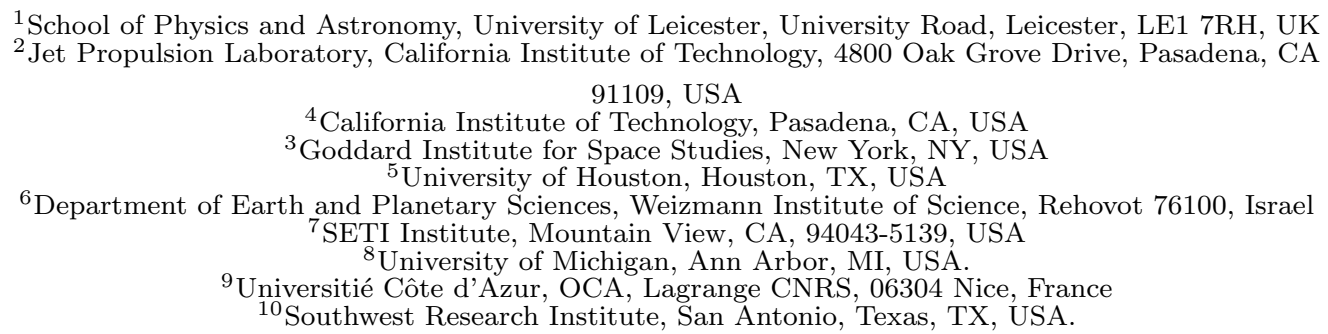

\section{Key Points:}

- Banded structure of Jupiter's microwave brightness is correlated with the cloudtop winds as far down as 100 bars.

- Belt/zone contrasts flip sign in the 5-10 bar region, a transition layer coinciding with the water condensation level.

- Transition can be explained by stacked meridional circulation cells and/or latitudinal gradients in precipitation. 

riers to meridional mixing (Read et al., 2006). Conversely, belts are warm (adiabatic compression) and feature westward (retrograde) jets on their poleward boundaries.

The upper-tropospheric belt/zone temperature contrasts encourage condensation of volatiles (e.g., ammonia) in cooler regions, typically producing reflective aerosols in zones and cloud-free conditions in belts, although the correspondence between the zonal jets and the opacity of the clouds (sensed at $5 \mu \mathrm{m}$, Antuñano et al., 2019) only really holds at low latitudes. Conversely, the correspondence between the observed cloud-tracked winds and upper tropospheric temperatures persists up to high latitudes near $\pm 60^{\circ}$ (Conrath \& Pirraglia, 1983; Flasar, 1986; Simon-Miller et al., 2006; Fletcher et al., 2016) and implies, via the thermal wind equation (Holton, 2004), that the zonal jets decay with altitude from the cloud-tops to the tropopause (Pirraglia et al., 1981; Conrath et al., 1990). The source of the dissipative mechanism causing this decay with height remains unclear and has never been directly observed, but could be related to wave or eddy stresses opposing the winds (Pirraglia, 1989; Orsolini \& Leovy, 1993). Finally, the latitudinal distribution of chemicals such as ammonia (Gierasch et al., 1986; Achterberg et al., 2006; de Pater et al., 2016; C. Li, Ingersoll, et al., 2017), phosphine (Fletcher et al., 2009; Giles et al., 2017; Grassi et al., 2020), and para-hydrogen (Conrath et al., 1998; Fletcher, de Pater, et al., 2017), combined with the observed temperature and aerosol distributions, suggest that the atmospheric circulation in the upper troposphere is dominated by rising motions over zones, zoneto-belt meridional transport at high altitude, and sinking over the belts. This is the "classical" picture of belt/zone circulation envisaged by Hess and Panofsky (1951) and Stone (1976), and is often likened to 'Hadley-like' circulations in the terrestrial atmosphere, whereby warm tropical air rises and moves poleward (a thermally-direct circulation), being deflected eastward by the Coriolis effect to generate sub-tropical jet streams.

Insights from Voyager, Galileo, and Cassini have challenged this conceptual picture, as reviewed by Fletcher et al. (2020). Lightning was detected as optical flashes (Little et al., 1999; Gierasch et al., 2000; Baines et al., 2007), and was found to be prevalent in the belts but either absent or obscured in the zones. This suggested moist air converging and rising in the belts, potentially in narrow convective plumes embedded within regions of net subsidence (Lunine \& Hunten, 1987; Ingersoll et al., 2000; Showman \& de Pater, 2005). Furthermore, cloud-tracking by Voyager (Ingersoll et al., 1981) and Cassini (Salyk et al., 2006) identified eddies converging and supplying momentum to the eastward jets, via a process analogous to Earth's Ferrel cells (Vallis, 2006). This forcing of the jets by flux convergence can be confined to shallow layers within the clouds and yet still produce jets that extend deep (Lian \& Showman, 2008). However, the forcing must be balanced by a compensating meridional flow, which has rising motions in belts, belt-to-zone meridional transport, and sinking over the zones. Such a belt/zone circulation is opposite to that postulated for the upper troposphere, and has led to a hypothesis of 'stacked circulation cells,' with deep Ferrel-like cells dominated by eddy-forcing of the zonal winds, and upper cells of eddy-dissipation and wind decay (Ingersoll et al., 2000; Showman \& de Pater, 2005; Fletcher et al., 2020), with a poorly defined transition somewhere within the 'weather layer' above the water clouds. Such counter-rotating stacked cells have been observed in numerical simulations with prescribed heating and eddy momentum fluxes (Yamazaki et al., 2005; Zuchowski et al., 2009), and general circulation models (GCMs) hint at changes to the magnitude of eddy-momentum flux convergence as a function of altitude (Young et al., 2018; Spiga et al., 2020).

Juno's exploration of Jupiter provides an opportunity to explore belt/zone contrasts below the cloud tops, and to test the stacked-cell hypothesis. Jupiter's winds have been found to extend to approximately $3000 \mathrm{~km}$ below the clouds (Kaspi et al., 2018; Guillot et al., 2018), to the level where Ohmic dissipation may become important 



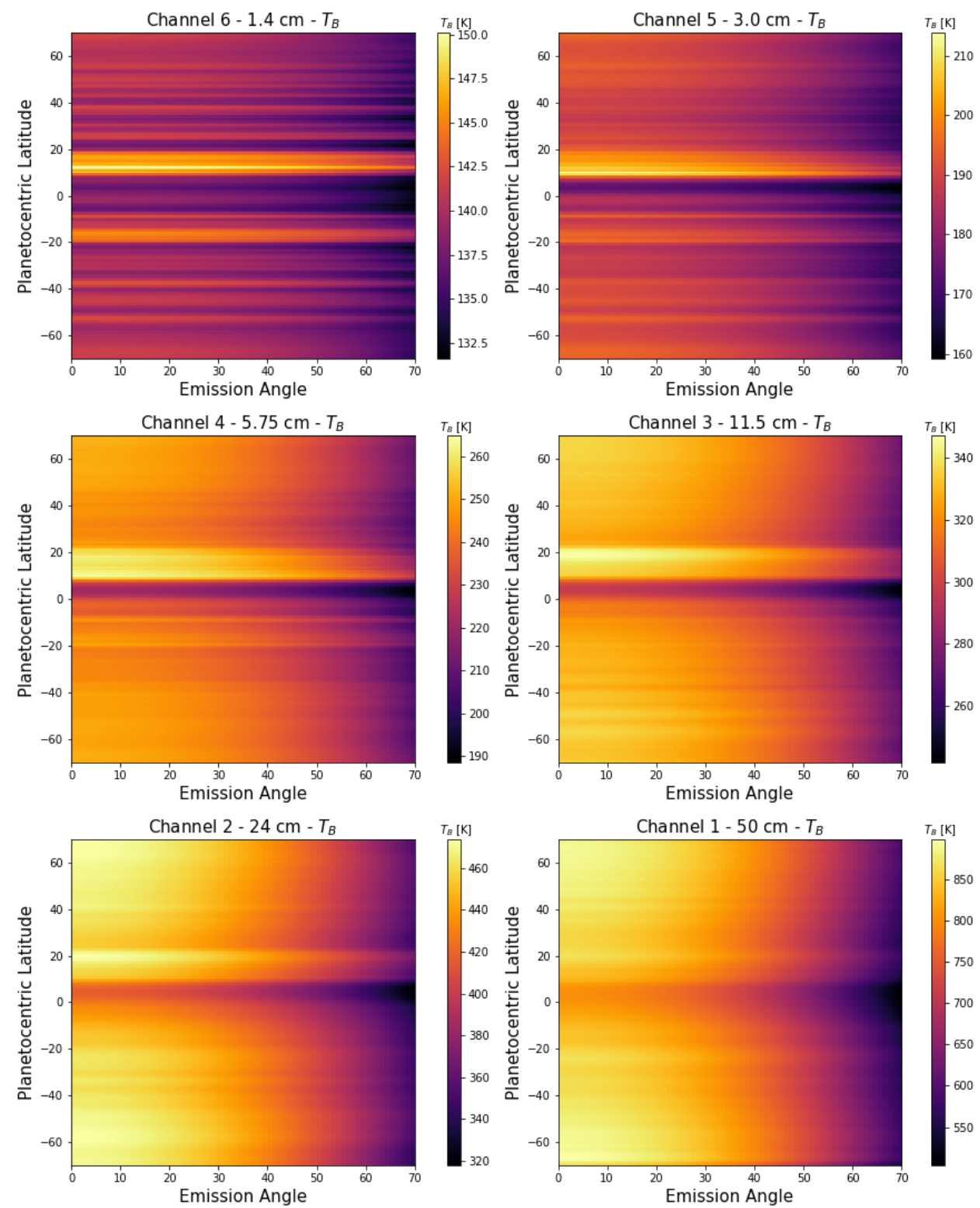

Figure 1. Deconvolved brightness temperatures as a function of emission angle and planetocentric latitude, formed from a weighted average of nine Juno perijoves between August 2016 and April 2018. Banded structure is observed in all channels, but the contrast is dominated by the tropics. No attempt has been made to remove the latitudinal dependence of $T_{B}$ on atmospheric scale height (which depends on effective gravitational acceleration), see Section 2.2. 


\subsection{Nadir Brightness Gradients}

Fig. 2 demonstrates how the filtering process of Oyafuso et al. (2020) identifies measurements that appear to differ substantially from other perjoves. For example, the microwave-bright southern periphery of the Great Red Spot was observed on PJ7 (C. Li, Oyafuso, et al., 2017) and is a significant outlier near $25^{\circ} \mathrm{S}$, but the poor goodness-of-fit $\left(\chi^{2}\right)$ for the quadratic in Eq. 1 for these latitudes means that PJ7 does not contribute significantly to our average. Similarly, a screening algorithm is used to remove observations contaminated by synchrotron emission, meaning that there will be fewer measurements available in affected latitudes for the quadratic fitting (see Section 2.5 of Oyafuso et al., 2020). This was particularly true for PJ3 and PJ4 at northern mid-latitudes, which appear anomalously bright but are constrained by very few uncontaminated measurements, such that their reduced weighting via the spatial contribution function minimises their contribution to the weighted average. The thick black line shows our best estimate of the microwave banding (consistent with Oyafuso et al., 2020), and is compared to the locations of the eastward (prograde, dashed) and westward (retrograde, dotted) jets as determined by Cassini/ISS cloudtracking of zonal winds $u$ (Porco et al., 2003), extracted via identifying locations where the vorticity $-\partial u / \partial y=0$ (where $y$ is the north-south distance in kilometres, accounting for the radius of curvature for an oblate spheroid). Similar calculations using Hubble cloud-tracked winds in 2017-19 are shown in the Supplementary Material, but the location of the jets has not changed significantly with time (Tollefson et al., 2017; Wong et al., 2020). We use these velocity minima and maxima to define the locations of Jupiter's cloud-top belts and zones, rather than the aerosol opacity, colour, and reflectivity, which are not good proxies for the underlying zonal wind structure (Fletcher et al., 2020).

To better emphasise the gradients observed by MWR, we convert the $T_{B}$ measurements into a 'pseudo-shear' $\Delta$ by analogy to the thermal wind equation (Holton, 2004), assuming constant pressure surfaces:

$$
\Delta=-\frac{g}{f T_{B}} \frac{\partial T_{B}}{\partial y}
$$

where we replace the kinetic temperature of the atmosphere with the brightness temperature. $f$ is the Coriolis parameter, $g$ is the gravitational acceleration at the particular pressure and latitude, and the brightness temperature derivative is evaluated on isobars (constant-pressure surfaces). At this stage, we make no connection between $\Delta$ and the shear on the zonal jets, but use this formalism simply to denote the edges of the microwave belts and zones. We plot $\Delta$ in Fig. 3, showing how the peaks in the microwave brightness gradients are co-located with the cloud-tracked zonal jets (the strength of the correlation will be explored below). Dashed lines are eastward jets (zones on the equatorward sides, belts on the poleward sides); dotted lines are westward jets (zones on the poleward side, belts on the equatorward side). Blue points are used to denote a negative gradient, red points are used for a positive gradient, and the patterns provide our first sign that a transition in belt/zone gradients occurs between the deep-sensing channels 1-3 (6 to greater than 100 bars), and the shallow-sensing channels $4-6$ ( 0.6 to 5.0 bars).

We can see this reversal in $\Delta$ by tracking single jets in Fig. 3. For example, the prograde jets at $48.6^{\circ} \mathrm{S}$ and $32.5^{\circ} \mathrm{S}$ coincide with local minima of negative $\Delta$ in the 0.6-5.0 bar range, but flip to being local maxima of positive $\Delta$ in the 10-100 bar range. Conversely, the retrograde jets at $35.5^{\circ} \mathrm{S}$ and $43.9^{\circ} \mathrm{S}$ coincide with local maxima of positive $\Delta$ at shallow depths, and local minima of negative $\Delta$ at deeper levels. This reversal in $\Delta$ has the effect of transitioning a traditional jovian belt (with prograde jets on their equatorward edges) from microwave-bright at shallow levels to microwave-dark at deeper levels, and vice versa for zones (with prograde jets on their poleward edges), as previously identified in PJ1 observations between $40^{\circ} \mathrm{S}$ and $40^{\circ} \mathrm{N}$ by Ingersoll et al. 

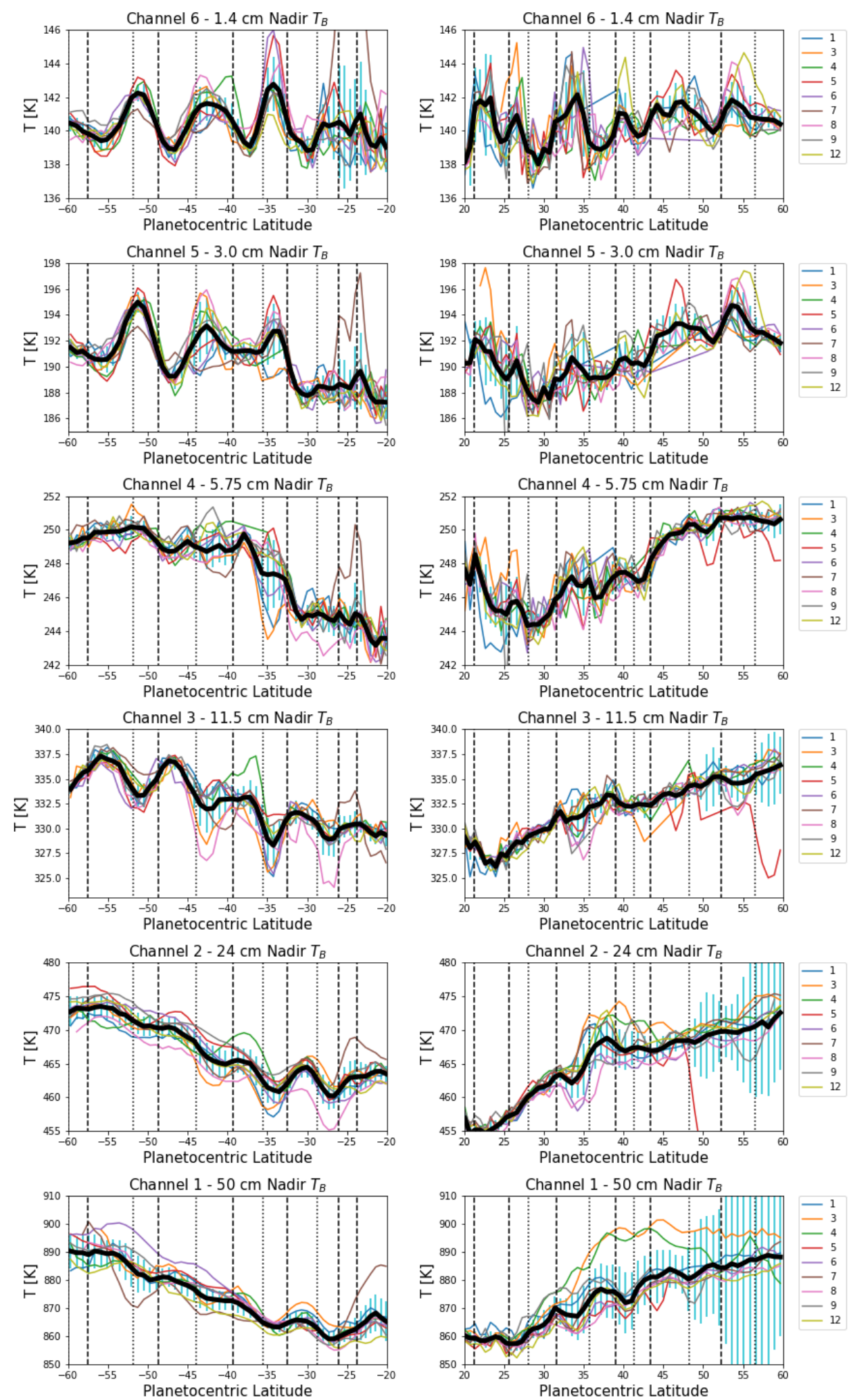

Figure 2. Nadir microwave brightness temperatures for all nine perijoves (coloured lines) compared to the weighted average (thick black line) to show the filtering process. Uncertainties on the weighted average are shown by the blue bars, indicating discrepancies between perijoves. These are compared to the peaks of eastward (dashed) and westward (dotted) zonal winds as measured by Cassini (Porco et al., 2003). Note that uncertainties become large at high northern latitudes for wavelengths longer than $11.5 \mathrm{~cm}$, due to the introduction of synchrotron noise into the beam. 

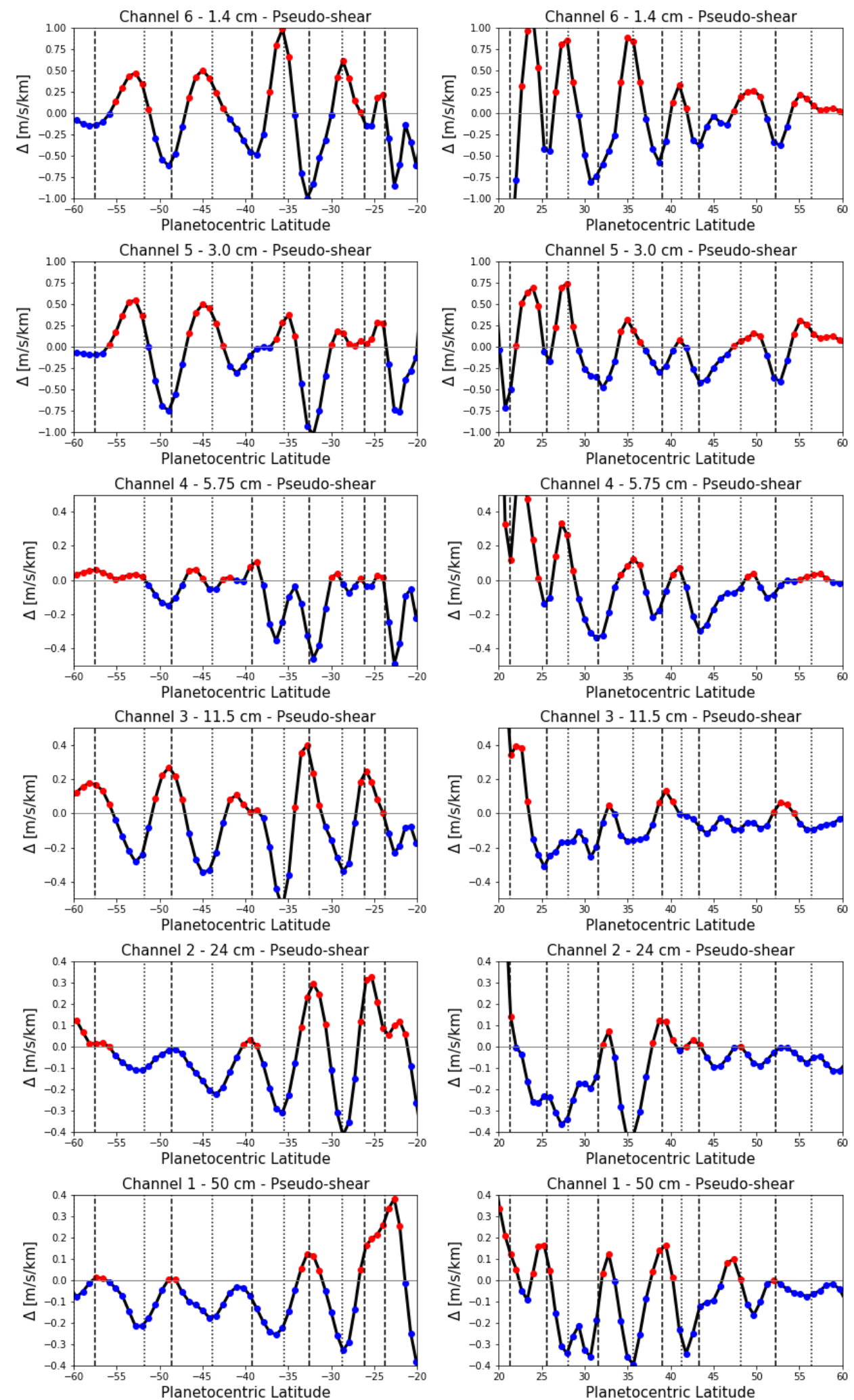

Figure 3. Nadir microwave brightness gradients for temperate latitudes, corrected by both the Coriolis parameter and gravitational acceleration to represent 'pseudo-shear' in m/s/km. Regions of negative pseudo-shear are represented by blue points, regions of positive pseudo-shear are represented by red points. These are compared to the peaks of eastward (dashed) and westward (dotted) zonal winds as measured by Cassini (Porco et al., 2003). 


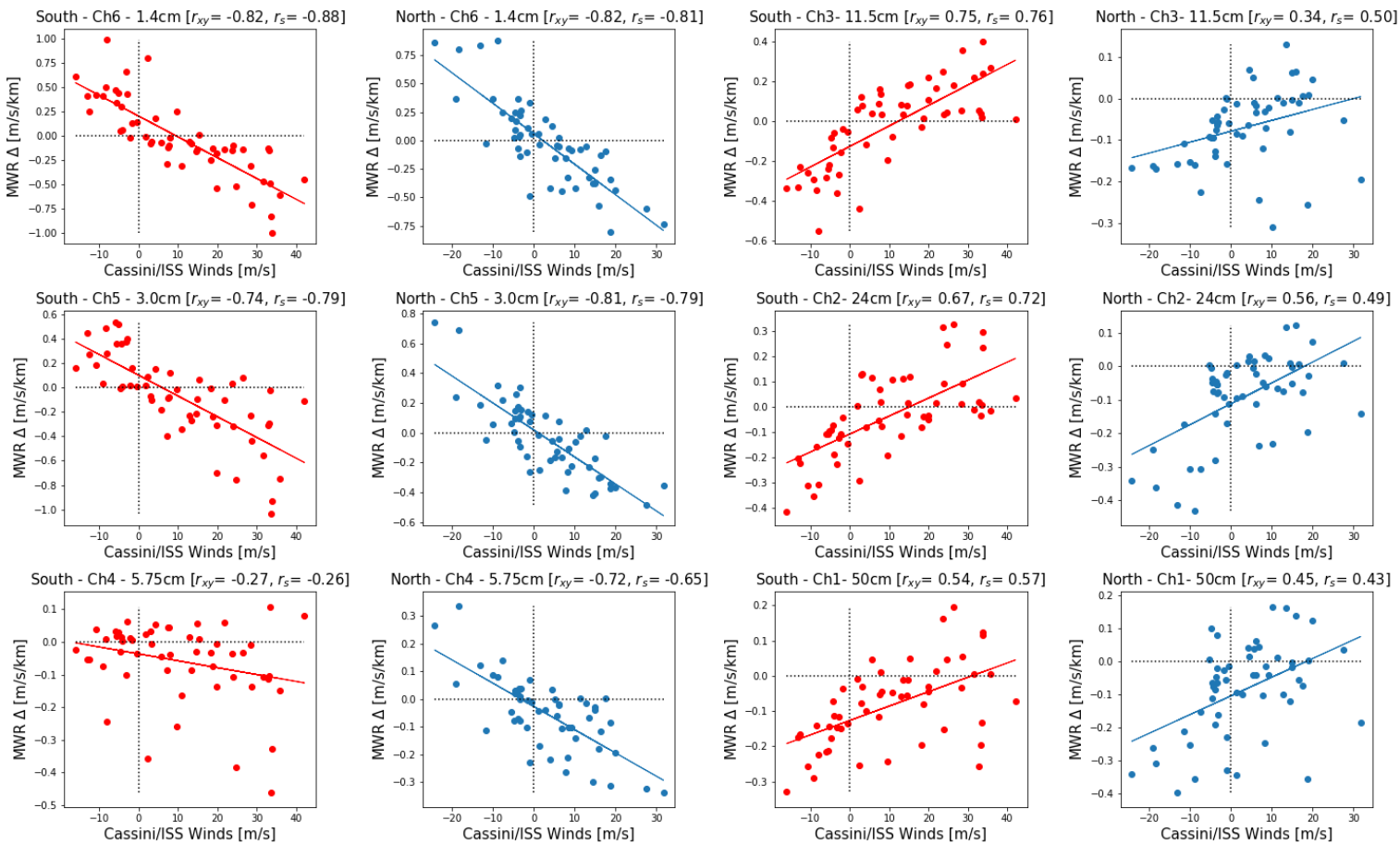

Figure 4. Scatter plots revealing negative (channels 4-6, left columns) and positive (channels 1-3, right columns) correlations between the nadir microwave $T_{B}$ gradients $\Delta$ and the Cassini cloud-tracked winds. Only latitudes between $25^{\circ}$ and $65^{\circ}$ in each hemisphere are included. Southern-hemisphere correlations are in red, northern-hemisphere correlations are in blue. A linear trend line has been added as a guide. The Pearson $r_{x y}$ and Spearman's ranked $r_{s}$ correlation coefficients are provided for each channel and hemisphere. See Supplementary Figures S1 and S2 for similar scatter plots computed using Hubble winds in 2017-19 (Wong et al., 2020; Tollefson et al., 2017). 

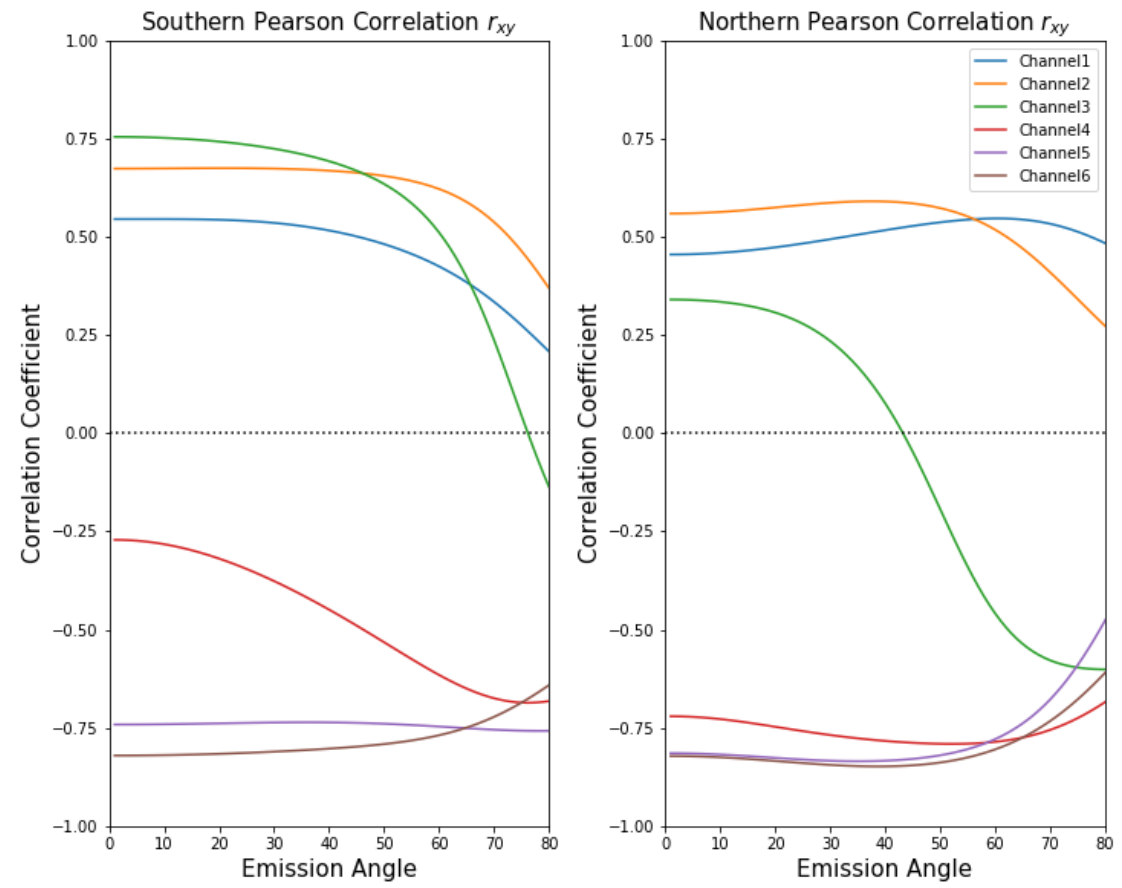

Figure 5. Linear correlation between microwave $T_{B}$ gradients $\left(\Delta_{\mu}\right)$ and cloud-top winds calculated on a $1^{\circ}$ grid at all emission angles (see Section 2.3 for a discussion of reliability at emission angles exceeding $\sim 60^{\circ}$ ). The channels naturally fall into two groups (positive and negative correlations), with a cross-over in Channel 3. These coefficients are hemispheric averages over the $25-65^{\circ}$ latitude ranges.

terials, Tables S1 and S2). Confirming the qualitative assessment in Fig. 4, p-values are smallest (and the correlation is highly statistically significant) for channel 5-6, and highest but still significant $(\sim 0.01)$ for channel 4 . We also computed these correlations using Hubble-derived zonal wind fields in 2017 (Tollefson et al., 2017) and 2019 (Wong et al., 2020), finding small improvements to the correlation without changing the conclusions - these computations can be found in our Supplementary Text S1.

The strength of the correlation depends on which perijoves are included in our weighted average, and which latitudes we include in the figure. In our Supplementary Text S2 we test the robustness of the correlations by selecting random pairs of perijoves from the nine studied here, recomputing the correlation coefficients and $p$-values for each pair and showing that the correlation remains significant, as it was when it was first noted in PJ1 data (August 2016) (Ingersoll et al., 2017; Oyafuso et al., 2020) - Figs. S4-S6. We also recomputed the correlation coefficients assuming winds that varied along cylinders parallel to the rotation axis (Duer et al., 2020), and found negligible changes to the strength of the correlations observed in Fig. 4.

Finally, we can extend the nadir-only analysis of Fig. 4 to all emission angles sampled by MWR, and represented by the limb-darkened brightness temperatures in Fig. 1 . We now calculate $\Delta_{\mu}$ for all $T_{B}(\phi, \mu)$ values (the $\mu$ subscript denotes that we now include all emission angles), and recompute the Pearson $r_{x y}$ in Fig. 5. The six channels still naturally fall into two groups - negative correlation at shallow depths, positive correlation at deeper levels. But Fig. 5 also shows that the transition from positive to negative correlation occurs within a single channel, channel $3(11.5 \mathrm{~cm})$, near $45^{\circ}$ emission angle in the north, and $75^{\circ}$ emission angle in the south, although 

Because of the enhanced $\mathrm{NH}_{3}$ retrieved in the Equatorial Zone, MWR channels tend to probe slightly higher in the equatorial region than they do in the neighbouring equatorial belts and the temperate mid-latitudes. For the right column of Fig. 6, we identify the pressure at the peak of the contribution function for each emission angle for six scenarios: three spatially averaged regions (northern mid-latitudes $20^{\circ} \mathrm{N}-40^{\circ} \mathrm{N}$, the equator $5^{\circ} \mathrm{N}-5^{\circ} \mathrm{S}$, and southern mid-latitudes $\left.20^{\circ} \mathrm{S}-40^{\circ} \mathrm{S}\right)$ and two different models of $\mathrm{NH}_{3}$ opacity - those of Hanley et al. (2009) and Bellotti et al. (2016). As we are primarily concerned with mid-latitudes in this study, we average the mid-latitude contribution functions for both opacity models and both hemispheres, and employ a quadratic spline fit to interpolate over the emission angles in our experiments. This provides smoothly varying functions for the angular dependence of the contribution functions at mid-latitudes, based on realistic $\mathrm{NH}_{3}$ abundances.

The calculations in Fig. 6 reveal that, between emission angles of $0^{\circ}$ and $70^{\circ}$, MWR sounds a range of pressures in each channel: $1.4 \mathrm{~cm}$ (channel 6, 0.55-0.64 bar), $3.0 \mathrm{~cm}$ (channel 5, 0.8-1.6 bar), $5.75 \mathrm{~cm}$ (channel 4, 2.3-4.8 bar), $11.5 \mathrm{~cm}$ (channel 3, 6.0-13.8 bar), $24 \mathrm{~cm}$ (channel 2, 17.7-34.4 bar) and $50 \mathrm{~cm}$ (channel 1, 44-117 bar). As expected, we find substantially less altitude sensitivity at the shortest wavelengths (channels 5 and 6 , sounding $p<2$ bar) compared to the highest wavelengths (channels 1 and 2 , sounding $p>20$ bar). This is consistent with the extent of the limb darkening shown in Fig. 1. We stress that the contribution functions remain extremely model dependent, varying with the retrieved ammonia abundances and assumptions about the lapse rate. Furthermore, the peaks represent broad functions, with extensions to lower and higher pressures, particularly at the longest wavelengths (Janssen et al., 2017). Channel $1(50 \mathrm{~cm})$ also displays significant sensitivity to pressures approaching 1000 bars, but this remains questionable given uncertainties about ammonia and water opacity at these long wavelengths (C. Li et al., 2020).

Based on the contribution functions in Fig. 6, we can approximate the depth of the $\Delta_{\mu}$ transition from Fig. 5 , where the flip from positive to negative correlations is observed in Channel $3(11.5 \mathrm{~cm})$. In the northern temperate domain this occurs near $\theta=40-50^{\circ}$ (Fig. 5), placing the transition near 10-11 bars. Similarly, the southern transition was at $\theta=70-80^{\circ}$, implying a transition nearer $4-6$ bars. These are averaged over all temperature latitudes in each hemisphere, and will be further refined below.

\subsection{Constructing a 2D Brightness Temperature Cross Section}

We now use the emission-angle dependence of the MWR contribution functions (Fig. 6) to assign the model-independent $T_{B}(\phi, \mu)$ measurements from Fig. 1 to a vertical pressure grid. We stress that this is a method for reprojecting the $T_{B}$ measurements onto a pressure grid using a model-dependent contribution function, and should not be confused with a full inversion of the measurements to derive real kinetic temperatures. This reprojection greatly expands the vertical sensitivity compared with the nadir-only approach, but we encounter substantial challenges, as shown in two example $T_{B}(p)$ profiles in Fig. 7. Firstly, the vertical sensitivity of adjacent MWR channels do not overlap with one another for emission angles smaller than $70^{\circ}$, so we are required to interpolate between them. Secondly, adjacent channels do not line up sufficiently to produce a completely smooth vertical structure, resulting in some kinks in the $T_{B}(p)$ profiles. This is particularly true for the transition between channels 5 and 6 , where there is an offset of tens of degrees. This is likely due to the assumptions underpinning the contribution function calculations: even though we have used realistic $\mathrm{NH}_{3}$ distributions, differences in the $\mathrm{NH}_{3}$ abundance could shift the peak sensitivity up and down and possibly allow better alignment of the channels. Thirdly, we are effectively treating the contribution function as a delta function, assigning the $T_{B}$ to a unique pressure level and ignoring the broad range of pressures sounded in Fig. 

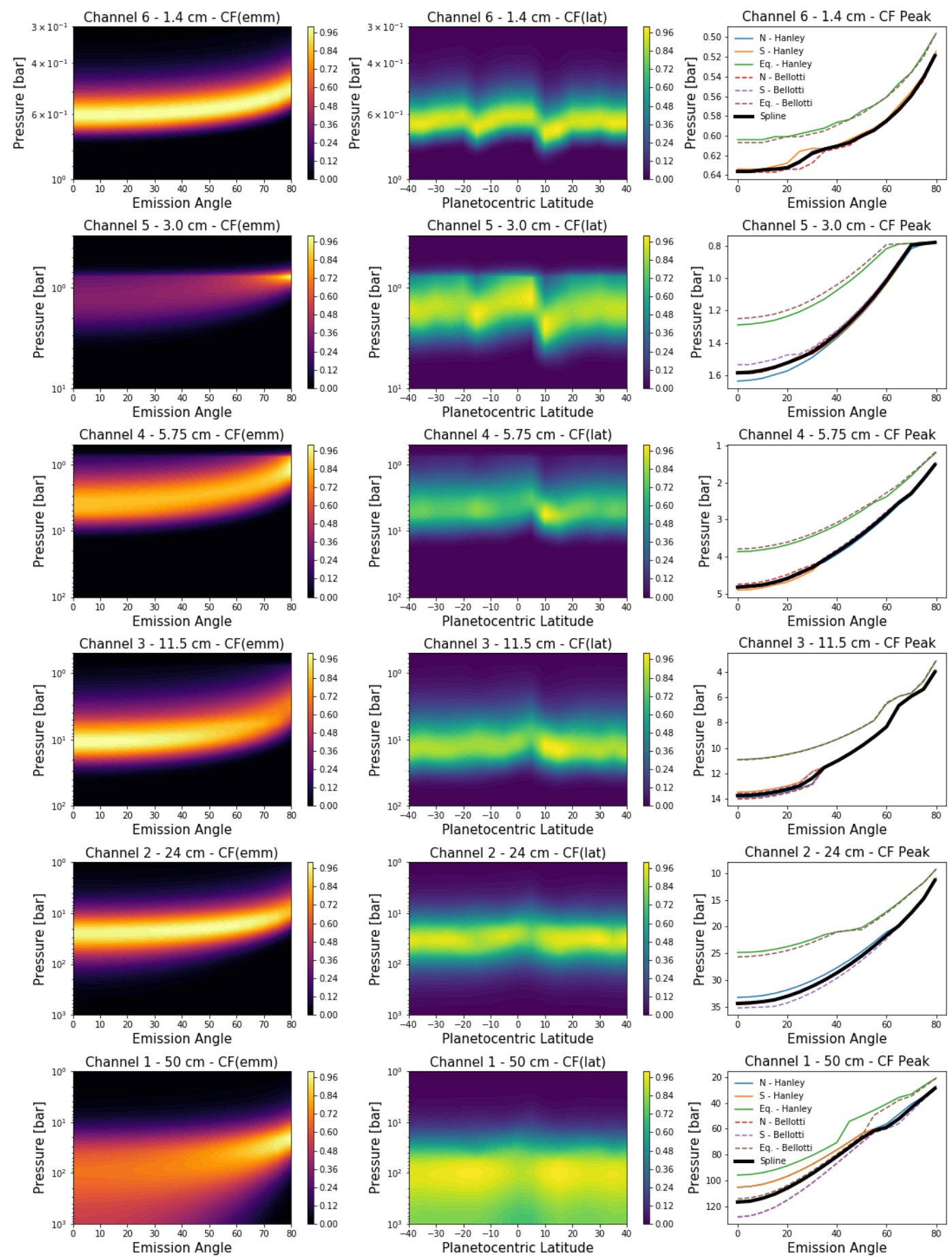

Figure 6. Contribution functions based on the retrieved distribution of $\mathrm{NH}_{3}$ versus latitude and pressure based on Guillot, Li, et al. (2020), with a modified $\mathrm{NH}_{3}$ gradient at $p<0.6$ bars to remove a discontinuity. Left: normalised contribution functions as a function of emission angle for the equator. Centre: normalised contribution functions at zero emission angle (nadir view) for all latitudes. Right: peak pressure of the contribution function averaged over three regions (north $20^{\circ} \mathrm{N}$ to $40^{\circ} \mathrm{N}$; south $20^{\circ} \mathrm{S}$ to $40^{\circ} \mathrm{S}$; and equator $5^{\circ} \mathrm{N}$ to $5^{\circ} \mathrm{S}$ ) using two different $\mathrm{NH}_{3}$ opacity models - Hanley et al. (2009) as the solid lines and Bellotti et al. (2016) as the dashed lines. The solid black line is the spline-interpolated contribution function described in the main text. 

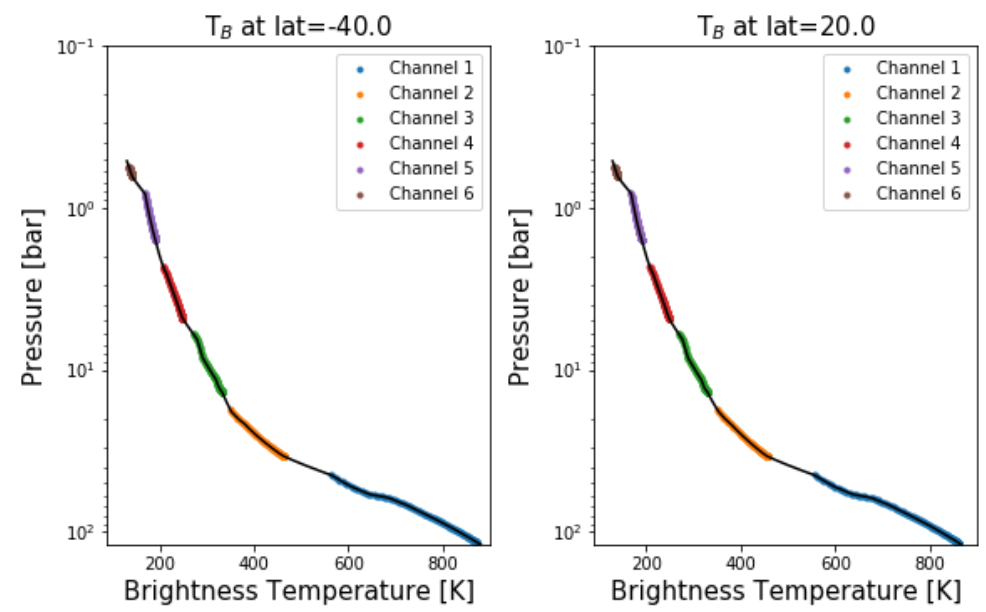

Figure 7. Vertical profiles of $T_{B}$ at two different latitudes, estimated by assigning limbdarkened MWR measurements to discrete pressure levels using the contribution function peaks in Fig. 6. The y-axis indicates the pressure of the contribution peak at different emission angles, and different colours indicate different channels, with a smooth interpolation over regions without MWR sensitivity (retaining emission angles smaller than $70^{\circ}$ ). Note that this is not from a spectral inversion, therefore does not represent kinetic temperatures - it is simply a reprojection of the MWR measurements.

6 - this will be particularly problematic for channel 1, which has a broad contribution function reaching pressures of 1000 bars or greater. And finally, the $T_{B}(\phi, \mu)$ has some dependence on the chosen functional form for the limb darkening (Eq. 1) for high emission angles $(\mu<0.6)$.

We construct $T_{B}(p)$ profiles for all latitudes and assemble them into a $T_{B}(\phi, p)$ cross section in Fig. 8, compared to the locations of the cloud-top zonal winds. Although this has the appearance of a kinetic temperature cross section common in atmospheric physics, we caution that these $T_{B}$ values are the product of both temperature and opacity variations. As for the nadir $T_{B}$ profiles in Fig. 2, the gradients away from the tropics are rather subtle, so we compute the 'pseudo-shear' $\Delta_{\mu}$ for every pressure level in Fig. 9a. Here, the transition from $\Delta_{\mu}>0$ (red) to $\Delta_{\mu}<0$ (blue), or vice versa, is visible throughout the temperate mid-latitudes (as well as the retrograde jets on the poleward edges of the NEB and SEB, discussed in Section 2.2).

The transition occurs where $\Delta_{\mu}=0$ and is evidently latitude-dependent, so we plot $\Delta_{\mu}$ for individual eastward and westward jets in Fig. 9b-c, highlighting the high degree of variability from jet to jet. The vertical trends in $\Delta_{\mu}$ are clearest for the broad westward jets, where Fig. 9c confirms that shears are generally positive for $p<10$ bars and negative for $p>10$ bars, although there is significant variability across the latitudes. However, for the eastward jets the picture is unclear - these are generally (but not always) experiencing negative $\Delta_{\mu}$ for $p<10$ bars, and they have small values $\left(\Delta_{\mu}< \pm 0.25 \mathrm{~m} / \mathrm{s} / \mathrm{km}\right)$ for $p>10$ bars, sometimes positive, sometimes negative. We show in Section 3.3 that this weak $\Delta_{\mu}$, if interpreted as real kinetic temperature contrasts, might imply that eastward jets largely remain eastward at all depths to 100 bars, whereas the westward jets with larger $\Delta_{\mu}$ variations can change direction with depth. The lack of clarity in $\Delta_{\mu}$ at the prograde jet locations could be a spatial-resolution effect related to their narrow or 'sharp' latitudinal widths, compared to the broad retrograde jets. Fig. 9 suggests that the transition typically occurs in 


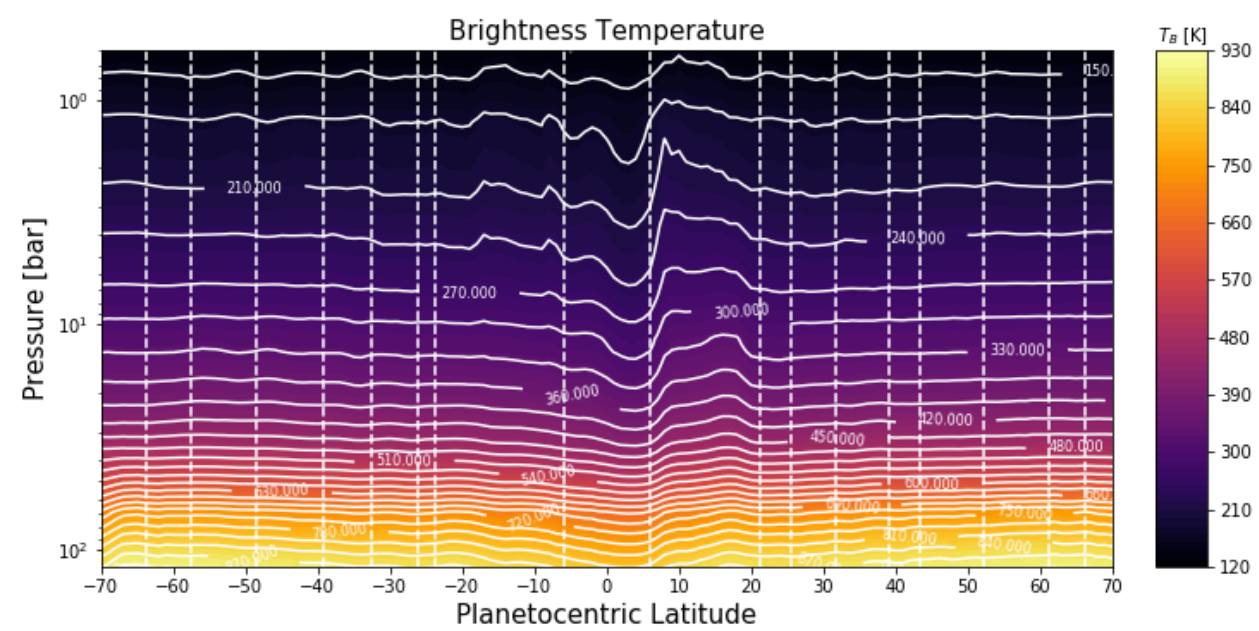

Figure 8. 2D cross-section of MWR brightness temperature $T_{B}(\phi, p)$, reprojected by assigning limb-darkened $T_{B}$ measurements to discrete pressure levels using the angular dependence of the contribution functions from Fig. 6. Vertical dashed lines indicate the locations of the cloud-top prograde jets.

the 5-10 bar range, and is certainly easier to see in the locations of the westward jets. In the next section, we explore what these pseudo-shears might imply about the zonal winds.

\subsection{Zonal Wind Interpretation}

\subsubsection{Dry Thermal Wind Balance}

Prior to this point, we have been careful to describe the microwave brightness contrasts in terms of a pseudo-shear, $\Delta$, because both opacity variations (mainly $\mathrm{NH}_{3}$ ) and kinetic temperature variations $(T)$ could be responsible for gradients in $T_{B}$. We now consider the extreme case where our measured $\Delta_{\mu}$ is assumed to be the true vertical windshear (i.e., that $T_{B}=T$, and that all brightness variations are considered to be due to kinetic temperature), and employ the 'dry' thermal wind equation (Holton, 2004), neglecting contributions from molecular weight gradients (see Section 3.3.4):

$$
\frac{\partial u}{\partial z} \approx-\frac{g}{f T}\left(\frac{\partial T}{\partial y}\right)_{p}
$$

Here $y$ is the north-south distance in kilometres, and the temperature gradients are measured on constant-pressure surfaces. We estimate the gravitational acceleration $g(p, \phi)$ using the combined gravitational and centrifugal potential of Buccino et al. (2020), reproducing their effective gravity at 1 bar. We then use the ideal gas law to estimate the height $z(p, \phi)$, which reproduces the altitudes recorded by the Galileo probe (Seiff et al., 1998). Both grids are provided with our Supplemental Material in Fig. S8.

We use Eq. 3 to integrate the cloud-top winds (Porco et al., 2003) as a function of depth. This quantity, the 'pseudo-wind,' is shown as a cross-section in Fig. 10b and for the individual jet locations in Fig. 10c-d. For simplicity, we integrate along the local vertical, rather than along cylinders parallel to the rotation axis, meaning that we cannot estimate winds close to the equator where the Coriolis parameter tends to zero. However, as we are dealing here with a relatively shallow layer of atmosphere, with a 
(a) Pseudo Shear $\Delta$

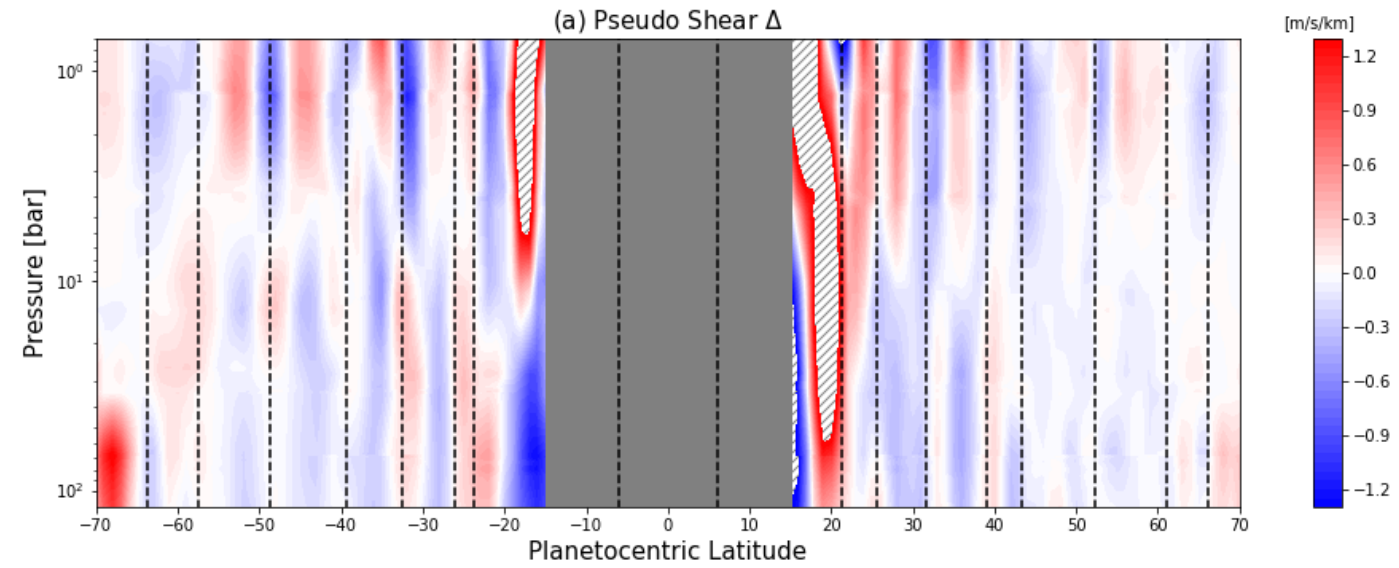

(b) Eastward Jet Shear
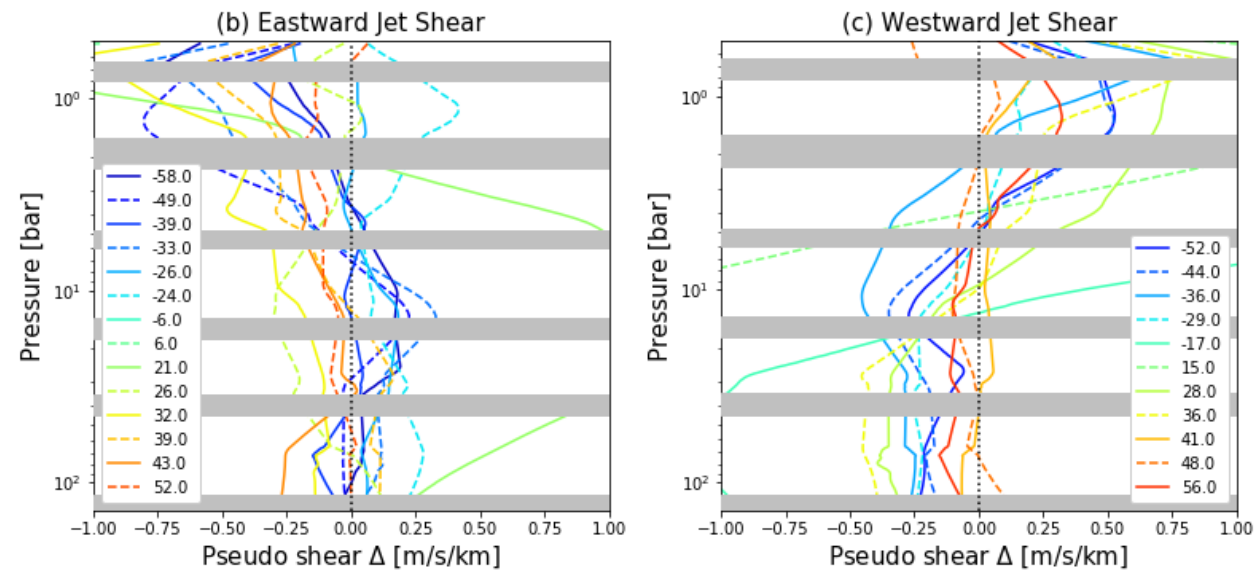

Figure 9. (a) 2D cross-section of MWR brightness gradient $\Delta_{\mu}(\phi, p)$, or pseudo shear, in units of $\mathrm{m} / \mathrm{s} / \mathrm{km}$, constructed from the $T_{B}(\phi, p)$ cross-section in Fig. 8. The colour scale is saturated at $\pm 1.3 \mathrm{~m} / \mathrm{s} / \mathrm{km}$ to emphasise gradients at mid-latitudes, values of $\Delta_{\mu}$ exceeding this range are shown as grey hatches. Tropical regions at latitudes less than $15^{\circ}$ are omitted. Vertical dashed lines indicate the locations of the cloud-top prograde jets. (b, c) Extracting the MWR pseudoshear $\Delta_{\mu}$ from (a) near to the locations of the eastward (b) and westward (c) jets, as shown by the planetocentric latitudes in the legends. Grey horizontal bars indicate regions without MWR vertical sensitivity (as defined by Fig. 6) and discontinuities in the calculation of $\Delta_{\mu}$. Tropical pseudoshears exceed $\pm 1 \mathrm{~m} / \mathrm{s} / \mathrm{km}$ over much of the domain, so cannot be seen on this figure. The pseudoshear generally reverses sign near the 10-bar level, especially for southernhemisphere jets. 

(a) Cloud-Tracked Winds [m/s]

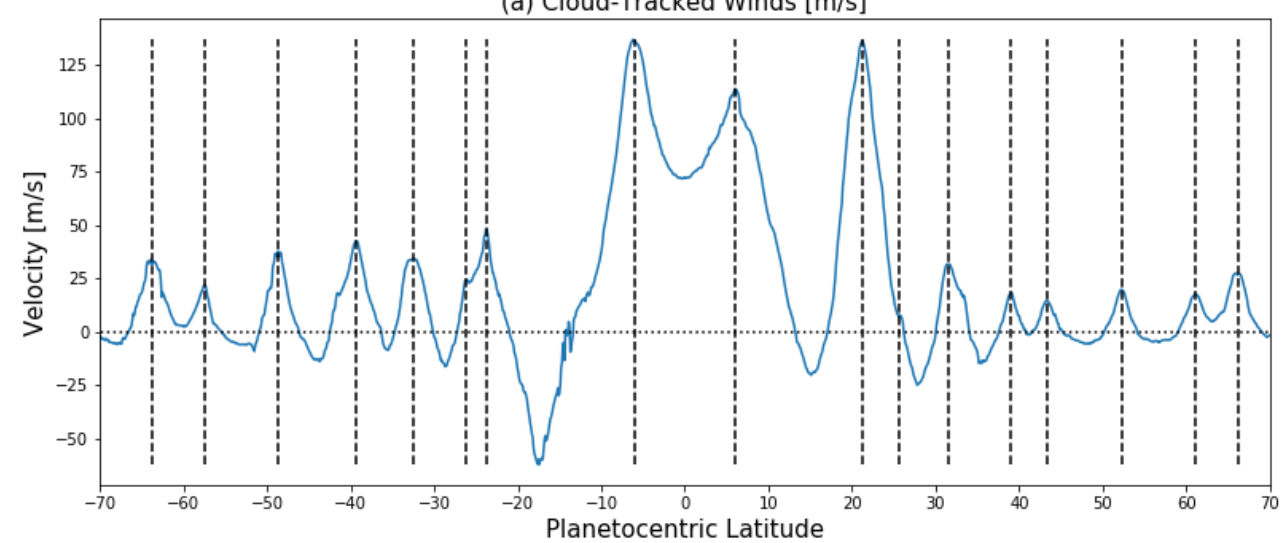

(b) Pseudo Zonal Wind

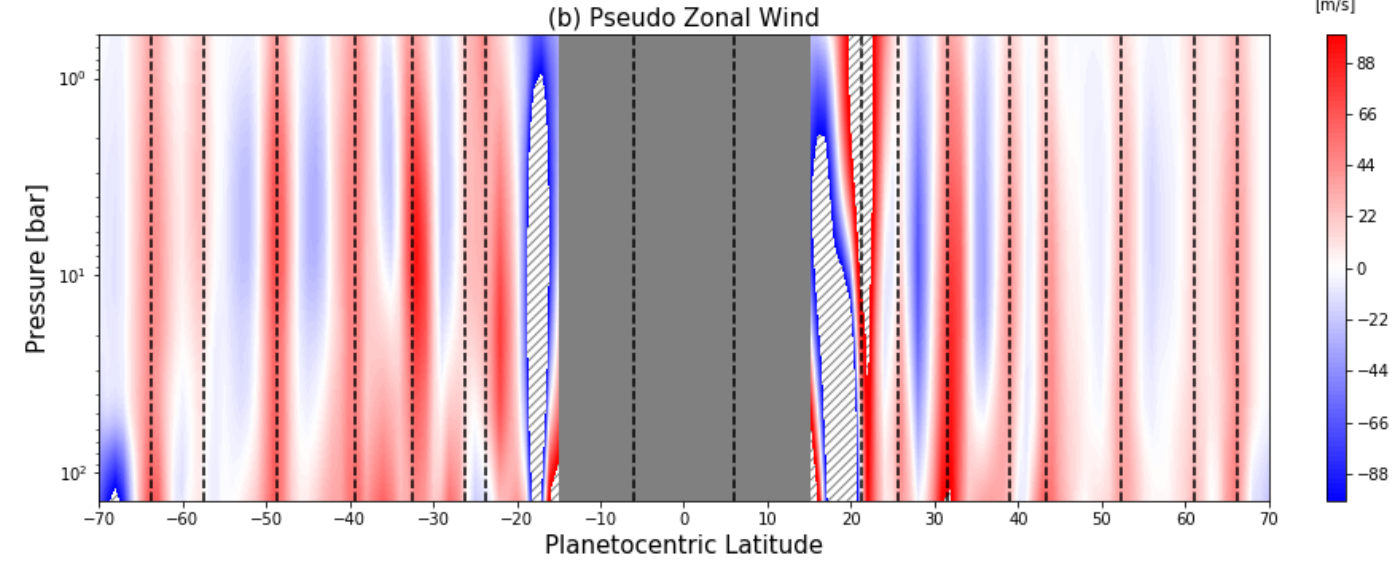

(c) Eastward Jets

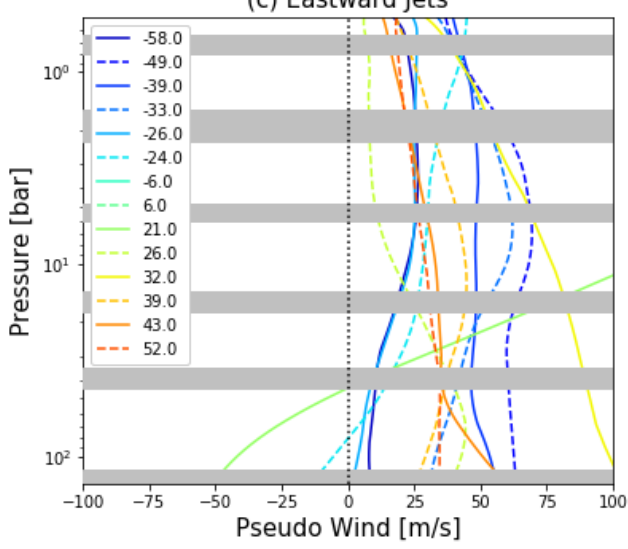

(d) Westward Jets

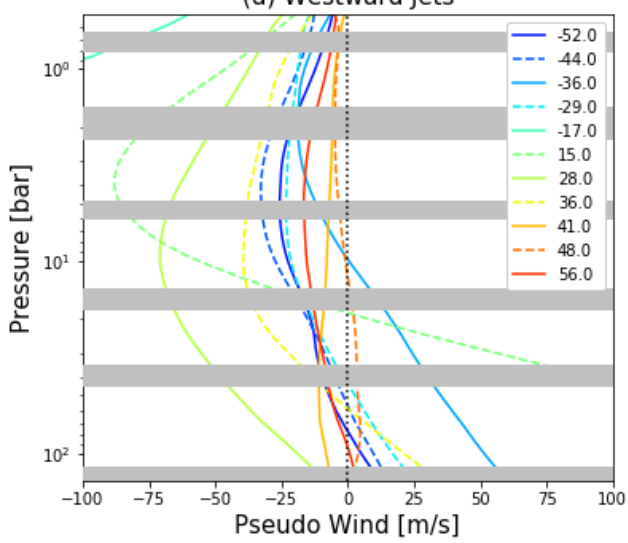

Figure 10. Calculated pseudo winds (b) assuming that $\Delta_{\mu}$ can be equated to the vertical shear on the zonal winds (i.e., that $T=T_{B}$ ). Integration is along the local vertical, rather than along cylinders parallel to the rotation axis. Cloud-tracked winds from Cassini (Porco et al., 2003)) are shown in panel (a) for comparison. Speeds exceeding $100 \mathrm{~m} / \mathrm{s}$ have been omitted (grey hatches), and speeds peak where $\Delta_{\mu}$ changes sign. Vertical dashed lines indicate the locations of the cloud-top prograde jets. Low latitudes near the equator are omitted as the Coriolis parameter tends to zero (it varies as the sine of the latitude) and $\Delta_{\mu}$ therefore tends to infinity. The lower panels show the MWR pseudowinds from (b), extracted near to the locations of the eastward (c) and westward (d) jets, as shown by the planetocentric latitudes in the legends. Grey horizontal bars indicate regions without MWR vertical sensitivity (as defined by Fig. 6) and discontinuities in the calculation of $\Delta_{\mu}$. Tropical windspeeds calculated in this manner exceed $\pm 100 \mathrm{~m} / \mathrm{s}$ over much of the domain, so cannot be seen on this figure. Note that this figure implies strengthening winds at $p>100$ bar, whereas Juno gravity measurements require that they must ultimately begin to decay at higher pressures (Kaspi et al-,21018). 

gradients along constant-pressure surfaces, we express the geostrophic thermal wind equation (Holton, 2004) in its less familiar 'moist' or 'virtual' form (sometimes known as a 'humidity wind' equation, Sun et al., 1991) in altitude coordinates $z$ :

$$
\frac{f T}{g} \frac{\partial u}{\partial z}=-\left(\frac{\partial T_{v}}{\partial y}\right)_{p}
$$

where symbols have the same meanings as in Section 3.3.1. Sun et al. (1991) demonstrated that compositional gradients could have a significant influence on the windshear in hydrogen-rich atmospheres, most important with the observed enrichments of Uranus and Neptune over solar composition, but here we explore the implications for Jupiter's troposphere. The virtual temperature $T_{v}$ is defined as:

$$
T_{v}=\frac{T}{1+\Sigma \alpha_{c} q_{c}}
$$

Here $q_{c}$ is the mole fraction, $\alpha_{c}$ is a coefficient for each constituent equal to $\left(\mu_{c} / \mu_{d}\right)-1$, the ratio of the molecular weight of the constituent $\left(\mu_{c}\right)$ to the molecular weight of dry air $\left(\mu_{d}\right)$. The $\Sigma$ symbol implies a sum over the relevant gases $\left(\mathrm{NH}_{3}, \mathrm{H}_{2} \mathrm{~S}, \mathrm{H}_{2} \mathrm{O}\right)$. We do not directly relate $T_{v}$ to the observed $T_{B}$ gradients, but introduce it simply to account for the effects of molecular weight gradients on vertical shears. The derivation below differs from Eq. 7 of Sun et al. (1991) because we use mole fractions, whereas they used mass mixing ratios. In the case where these constituents are considered to be variable, we adjust the thermal wind equation to become:

$$
\begin{array}{r}
\frac{f T}{g} \frac{\partial u}{\partial z}=-\frac{\partial}{\partial y}\left(\frac{T}{1+\Sigma \alpha_{c} q_{c}}\right) \\
=-\frac{1}{1+\Sigma \alpha_{c} q_{c}}\left(\frac{\partial T}{\partial y}-\frac{T}{1+\Sigma \alpha_{c} q_{c}} \frac{\partial}{\partial y}\left(\Sigma \alpha_{c} q_{c}\right)\right)
\end{array}
$$

If we retain the molecular weight contributions of all three condensables, but assume that both $\mathrm{H}_{2} \mathrm{~S}$ and $\mathrm{H}_{2} \mathrm{O}$ are latitudinally uniform to remove their derivatives, then we can rewrite the $T_{v}$ gradient considering only contributions from the temperature and $\mathrm{NH}_{3}$ gradients:

$$
\frac{f T}{g} \frac{\partial u}{\partial z}=-\frac{1}{1+\Sigma \alpha_{c} q_{c}}\left(\frac{\partial T}{\partial y}-\frac{T \alpha_{N H_{3}}}{1+\Sigma \alpha_{c} q_{c}} \frac{\partial q_{N H_{3}}}{\partial y}\right)
$$

In the case where we assume no latitudinal ammonia gradients, and with $\Sigma \alpha_{c} q_{c}<<$ 1 (a reasonable assumption in the upper troposphere where mole fractions of each species are $<10^{-3}$, but more questionable at depth), this simplifies to the familiar dry thermal wind equation in Eq. 3, as discussed in Section 3.3.1. However, if we assume negligible latitudinal contrasts in temperature, following previous MWR analyses (C. Li, Ingersoll, et al., 2017; Ingersoll et al., 2017), and again assuming $\Sigma \alpha_{c} q_{c}<<1$, then we find that ammonia gradients can still result in vertical windshear:

$$
\frac{\partial u}{\partial z} \approx+\frac{g \alpha_{N H_{3}}}{f} \frac{\partial q_{N H_{3}}}{\partial y}
$$

Here $\alpha_{N H_{3}}=\left(\mu_{N H_{3}} / \mu_{d}\right)-1=6.36$, with $\mu_{N H_{3}}=17.031 \mathrm{~g} / \mathrm{mol}$ and the dry molecular weight of jovian air is $\mu_{d} \approx 2.313 \mathrm{~g} / \mathrm{mol}$, assuming $86.26 \% \mathrm{H}_{2}, 13.54 \% \mathrm{He}$, and $0.20 \%$ $\mathrm{CH}_{4}$ (von Zahn et al., 1998; Wong et al., 2004). Note the change in sign between the two forms of the wind equation (Eq. 3 and 9), and how it relates to the MWR brightness temperature observations. Local maxima in microwave brightness over belts in the upper troposphere $(p<5$ bar) would still be in balance with negative $\partial u / \partial z$ (i.e., wind decay with height) irrespective of whether this is due to an increased temperature or an $\mathrm{NH}_{3}$ minimum. Local minima in $T_{B}$ in the deeper troposphere $(p>10$ bar $)$ would still be in balance with positive $\partial u / \partial z$ (i.e., wind decay with depth), irrespective of 


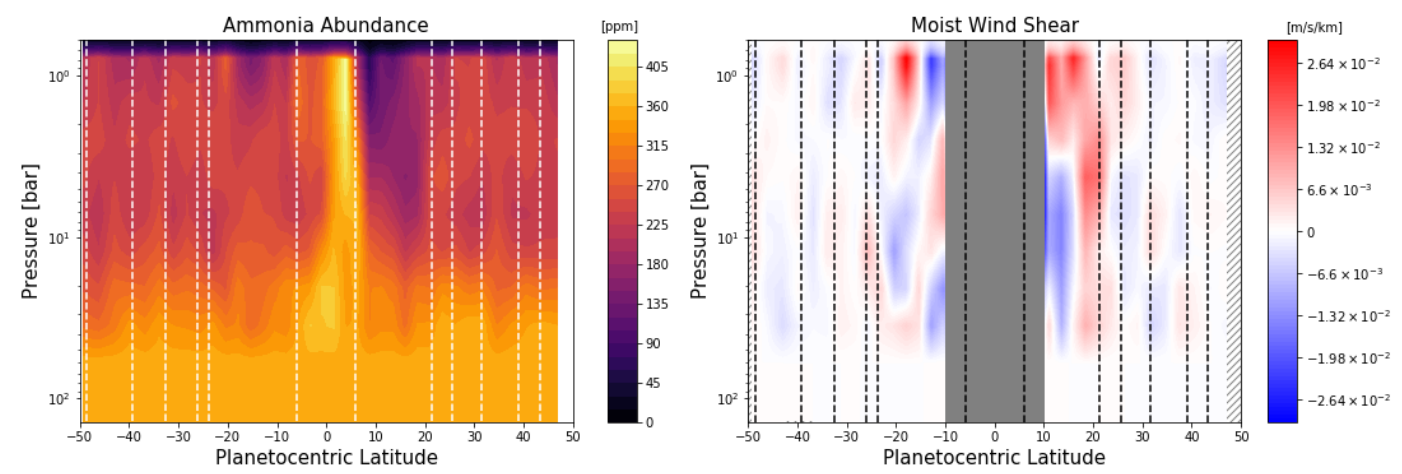

Figure 11. Zonal-mean cross section of ammonia derived by Guillot, Li, et al. (2020) based on the technique of C. Li, Ingersoll, et al. (2017). The gradients are used to estimate the moist shear based on $\mathrm{NH}_{3}$ alone, which is some $50 \times$ smaller than that in Fig. 9 for mid-latitudes.

constraints on deep kinetic temperatures are sorely needed, as we explore in the next section.

\subsubsection{Deep Thermal Contrasts}

Breaking the degeneracy between deep temperature and ammonia contrasts via remote sensing alone (e.g., microwave and infrared) remains a challenge. However, we can gain insights on the likelihood of deep temperature gradients (and winds that increase in speed from the cloud tops to the 5-10-bar level) by (i) considering the stability of the zonal wind solutions; and (ii) exploring the results of deep convection models.

For the former, the top-down constraint on the jet structure offered by vorticity measurements support the suggestion that the winds must increase with depth from the cloud-tops to regions near the water cloud (Dowling, 1995). As the meridional gradient of the potential vorticity changes sign at multiple locations (e.g., Read et al., 2006), the cloud-top winds (and our inferred winds at depth) have multiple critical latitudes which could be stable, unstable, or neutrally stable (Dowling, 1995, 2020). Before the descent of the Galileo probe, Dowling (1995) used Voyager-era vorticity measurements (Limaye, 1986) and a shear-stability analysis to determine Jupiter's deep wind profile in the 5-8 bar region. To make the cloud-top critical latitudes stable, rather than marginally stable, required an increase in the amplitude of the underlying eastward jets compared to the cloud-top jets by a factor of approximately two, with larger changes at lower latitudes than at mid-latitudes. The magnitude of the change depended on the first-baroclinic deformation length, $L_{d}$, which remains rather uncertain at depth. Their suggested negative vertical shear of the zonal winds between the tropopause and the 5-8 bar level was later shown to be consistent with Galileo probe results (Atkinson et al., 1998), and qualitatively supports our suggestion that winds strengthen between the cloud-tops and the jovicline in the upper cell (i.e., that kinetic temperatures must vary with latitude, helping to explain the negative pseudoshear in shallow-sounding MWR channels 4-6).

Finally, deep-shell models of turbulent convection in rapidly-rotating fluid planets produce nested cylindrical flows aligned with the rotation axis, with alternating zonal jet structures and associated meridional temperature contrasts (Aurnou et al., 2008; Heimpel et al., 2016). These models produce axial thermal plumes parallel to the rotation axis, with the jets acting as barriers to cylindrically radial heat transfer. 

(a) Meridional Circulation Cells

(b) Mushball Precipitation

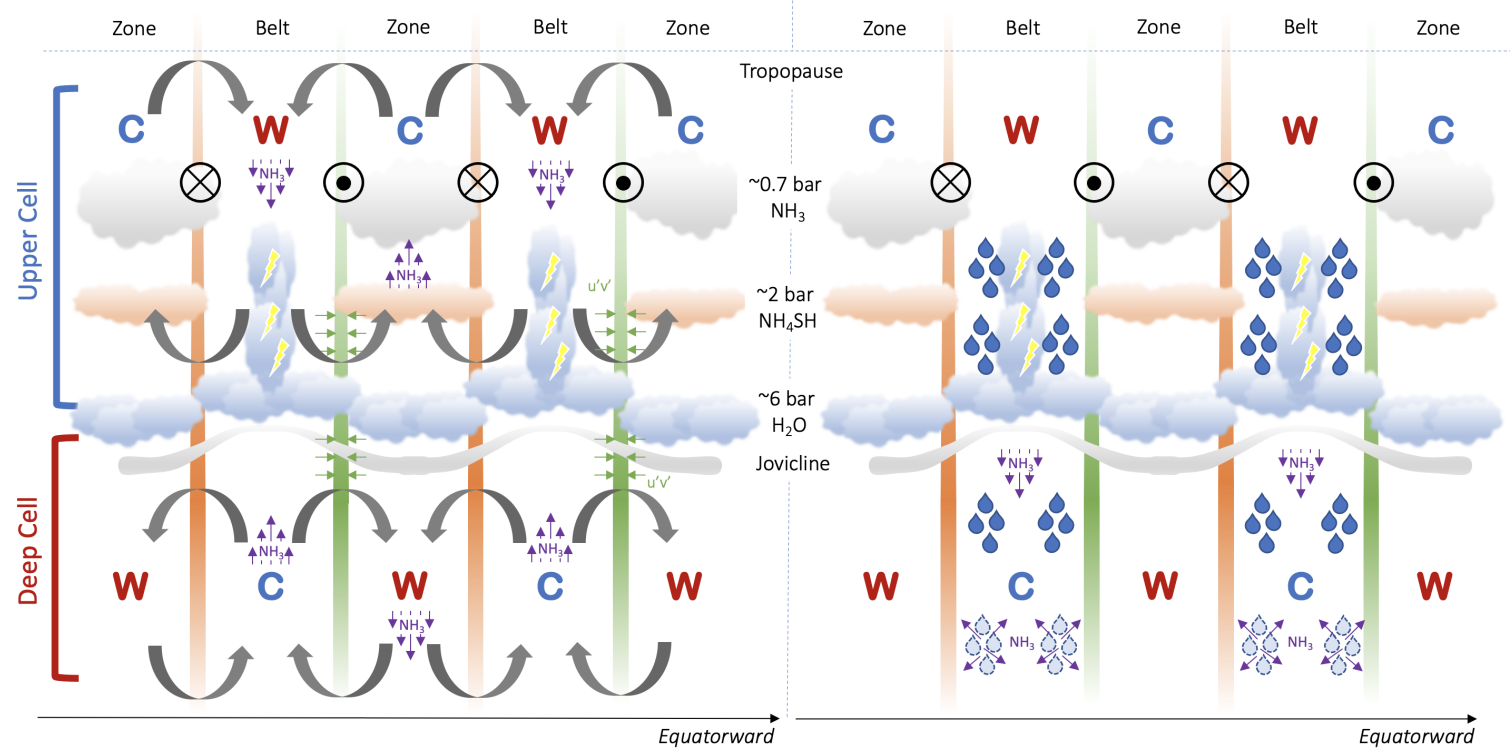

Figure 12. Conceptual diagrams of (a) the stacked system of meridional cells (adapted from Showman \& de Pater, 2005; Fletcher et al., 2020); and (b) mushball precipitation (Guillot, Li, et al., 2020). We stress that reality is likely to combine both of these concepts, and all altitudes are qualitative. In both diagrams, high microwave brightness is denoted by a red 'W' (warm), low microwave brightness is denoted by a blue ' $\mathrm{C}$ ' ( $\mathrm{cool})$; storm plumes are indicated as rising clouds with lightning flashes. The equator is to the right, such that belts have prograde jets on their equatorward edges. Eastward prograde jets are green (with a circular dot indicating motion out of the page) with eddy-momentum flux convergence (small green arrows); westward retrograde jets are orange (with a circular cross indicating motion into the page). The colouration of the green and orange bars indicate wind strengthening through the upper cell and wind decay with depth in the deep cell ('dry convective layer'). The jovicline is shown in grey, co-located with the stable stratification of the water cloud. Purple arrows indicate general ammonia depletion or enrichment, either as a consequence of meridional circulation (grey curved arrows, left) or as a consequence of sequestration in 'mushballs', precipitation, and re-evaporation at great depth (droplets, right), leading to steep vertical $\mathrm{NH}_{3}$ gradients in the belts.

\subsection{Stacked Meridional Circulation Cells}

As described in Section 1, the concept of multiple tiers of stacked circulation cells (Ingersoll et al., 2000; Showman \& de Pater, 2005; Fletcher et al., 2020) has been used as a possible resolution to the discrepancy between (i) zone-to-belt transport and subsidence in belts above the clouds inferred from Jupiter's upper tropospheric temperatures and composition; and (ii) belt-to-zone transport in Ferrel-like cells below the clouds and upwelling in belts inferred from the prevalence of lightning in Jupiter's belts (Ingersoll et al., 2000) and the meridional flow required to balance the eddymomentum flux convergence on the prograde jets (Fig. 12a). The change in the microwave brightness contrast across the transition would be consistent with $\mathrm{NH}_{3}$ (and potentially other gaseous species) being locally depleted in belts in the upper tier, and locally enhanced in belts in the deeper tier (Showman \& de Pater, 2005; Ingersoll et al., 2017). The transition between these tiers was assumed to exist somewhere 

The observed cloud-top winds could therefore be an underestimate of the maximum windspeeds in the upper troposphere (Fig. 10b).

However, this contrived picture is incomplete - it does not explain the extreme ammonia enrichment at the equator, nor does it explain why the global-scale $\mathrm{NH}_{3}$ depletion appears to extend to the 40-60 bar level (Ingersoll et al., 2017; C. Li, Ingersoll, et al., 2017), far deeper than simple precipitation might suggest (e.g., via the inclusion of ammonia rain, C. Li \& Chen, 2019). Ferrel-like circulation cells below the jovicline (Showman \& de Pater, 2005; Young et al., 2018), balancing eddy-momentum flux convergence on the prograde jets (Salyk et al., 2006), could extend deep even if the forcing is shallow (Lian \& Showman, 2008), driving temperature and compositional variability at tens of bars. The belt/zone meridional circulations inferred here may be superimposed onto this larger-scale structure (equatorial $\mathrm{NH}_{3}$ enrichment, midlatitude $\mathrm{NH}_{3}$ depletion) driven by precipitation, to be explored in the next section. Lightning could still be prevalent in the belts in Fig. 12a with this deeper jovicline, if rising motion from the deep 'dry-convecting' layer provides the initial instability to initiate buoyant moist convection and lightning in the water-cloud layers and above (Dowling \& Ingersoll, 1989; Thomson \& McIntyre, 2016). This could work if the stably-stratified transition zone were thinner (and easier to overcome) in the belts compared to the zones - a possible consequence of winds that decay with depth into the deeper layers (Thomson \& McIntyre, 2016).

\subsection{Precipitation and Microwave Brightness}

The complexity of the stacked-cells hypothesis may yet be its undoing, so we should ask whether vertical and meridional motions are truly required to explain the transition in the microwave belt/zone contrasts. Recent work by Guillot, Stevenson, et al. (2020) suggested that partially-melted hailstones of ammonia dissolved in water ice (nicknamed 'mushballs') could form at 1-2 bar when water is lofted upwards during powerful storms (this is also the level of shallow lightning flashes recently discovered by Juno, Becker et al., 2020). These mushballs then fall deep below the expected water cloud (Fig. 12b), to 5-30 bar depending on their properties and the available water ice, where they evaporate, causing cold and volatile-rich evaporative downdrafts that further deplete the condensates. Guillot, Stevenson, et al. (2020) use this process to explain the observed deep depletion of $\mathrm{NH}_{3}$ down to the $20-30$ bar region $(\mathrm{C} . \mathrm{Li}$, Ingersoll, et al., 2017; Ingersoll et al., 2017).

As storms are more prevalent within Jupiter's belts, we might expect $\mathrm{NH}_{3}$ depletion in the upper troposphere to be strongest here (producing the microwave-bright belts for $p<5$ bars). Similarly, as the mushballs evaporate to relinquish their ammonia (and water), they increase the mean molecular weight in the deeper troposphere, and generate cool downdrafts (Sugiyama et al., 2014). This could lead to a localised $\mathrm{NH}_{3}$ enhancement in the belts at depth (i.e., microwave-dark belts at $p>10$ bars). Combined, this leads to a steep $d q_{\mathrm{NH}_{3}} / d z$ gradient in the belts, shown in Fig. 12b, as precipitation dominates over any upward mixing. Conversely, Guillot, Li, et al. (2020) suggested that the absence of storms and mushballs in the Equatorial Zone was responsible for the vertical homogeneity of the $\mathrm{NH}_{3}$ distribution there. Here we suggest that a shallow $d q_{N H_{3}} / d z$ gradient could also persist in the extratropical zones for the same reason (i.e., upward mixing dominates over precipitation), providing the contrast to the larger $d q_{N_{3}} / d z$ in the stormy belts. At high pressures, slow horizontal mixing would serve to transport $\mathrm{NH}_{3}$ from belts into zones, and vice versa at lower pressures.

Guillot, Li, et al. (2020) parameterised the storm frequency using the MWR observations of Brown et al. (2018) - however, the detection of lightning sferics in the microwave still placed non-negligible storm flashes in regions considered as zones, and an imperfect relationship between local maxima in the storm rates and the location 


Planetary Data System Atmospheres Node https://pds-atmospheres.nmsu.edu/ data_and_services/atmospheres_data/JUNO. Data for individual figures are available through Zenodo https://doi.org/10.5281/zenodo.4740711.

\section{References}

Achterberg, R. K., Conrath, B. J., \& Gierasch, P. J. (2006). Cassini CIRS retrievals of Ammonia in Jupiter's Upper Troposphere. Icarus, 182, 169-180. doi: 10 .1016/j.icarus.2005.12.020

Allison, M. (1990, February). Planetary waves in Jupiter's equatorial atmosphere. Icarus, 83, 282-307. doi: 10.1016/0019-1035(90)90069-L

Allison, M., \& Atkinson, D. H. (2001). Galileo Probe Doppler residuals as the wave-dynamical signature of weakly stable, downward-increasing stratification in Jupiter's deep wind layer. Geophys. Res. Lett., 28, 2747-2750. ～doi: 10.1029/2001GL012927

Antuñano, A., Fletcher, L. N., Orton, G. S., Melin, H., Milan, S., Rogers, J., .. . Giles, R. (2019, Sep). Jupiter's Atmospheric Variability from Long-term Ground-based Observations at $5 \mu \mathrm{m}$. Astronomical Journal, 158(3), 130. doi: $10.3847 / 1538-3881 / \mathrm{ab} 2 \mathrm{~cd} 6$

Atkinson, D. H. (2001, September). The GalileoJupiter Probe Doppler Wind Experiment. Solar System Research, 35(5), 354-375.

Atkinson, D. H., Pollack, J. B., \& Seiff, A. (1998, September). The Galileo probe Doppler wind experiment: Measurement of the deep zonal winds on Jupiter. $J$. Geophys. Res., 103, 22911-22928. doi: 10.1029/98JE00060

Atreya, S. K., Wong, M. H., Owen, T. C., Mahaffy, P. R., Niemann, H. B., de Pater, I., ... Encrenaz, T. (1999). A comparison of the atmospheres of Jupiter and Saturn: deep atmospheric composition, cloud structure, vertical mixing, and origin. Plan. 6 Space Sci., 47, 1243-1262.

Aurnou, J., Heimpel, M., Allen, L., King, E., \& Wicht, J. (2008, June). Convective heat transfer and the pattern of thermal emission on the gas giants. Geophysical Journal International, 173(3), 793-801. doi: 10.1111/j.1365-246X.2008 $.03764 . \mathrm{x}$

Baines, K. H., Simon-Miller, A. A., Orton, G. S., Weaver, H. A., Lunsford, A., Momary, T. W., ... Ressler, M. E. (2007, October). Polar Lightning and Decadal-Scale Cloud Variability on Jupiter. Science, 318, 226-228. doi: 10.1126/science.1147912

Becker, H. N., Alexander, J. W., Atreya, S. K., Bolton, S. J., Brennan, M. J., Brown, S. T., ... Steffes, P. G. (2020, August). Small lightning flashes from shallow electrical storms on Jupiter. $\quad$ Nature, 584(7819), 55-58. $\quad$ doi: 10.1038/s41586-020-2532-1

Bellotti, A., Steffes, P. G., \& Chinsomboom, G. (2016, December). Laboratory measurements of the $5-20 \mathrm{~cm}$ wavelength opacity of ammonia, water vapor, and methane under simulated conditions for the deep jovian atmosphere. Icarus, 280, 255-267. doi: 10.1016/j.icarus.2016.07.013

Bolton, S. J., Adriani, A., Adumitroaie, V., Allison, M., Anderson, J., Atreya, S., ... Wilson, R. (2017, May). Jupiter's interior and deep atmosphere: The initial pole-to-pole passes with the Juno spacecraft. Science, 356, 821-825. doi: $10.1126 /$ science.aal2108

Brown, S., Janssen, M., Adumitroaie, V., Atreya, S., Bolton, S., Gulkis, S., ... Connerney, J. (2018, Jun). Prevalent lightning sferics at 600 megahertz near Jupiter's poles. Nature, 558, 87-90. doi: 10.1038/s41586-018-0156-5

Buccino, D. R., Helled, R., Parisi, M., Hubbard, W. B., \& Folkner, W. M. $\quad$ (2020, August). Updated Equipotential Shapes of Jupiter and Saturn Using Juno and Cassini Grand Finale Gravity Science Measurements. Journal of Geophysical Research (Planets), 125(8), e06354. doi: 10.1029/2019JE006354 
Cao, H., \& Stevenson, D. J. $\quad$ (2017, November). Z Zonal flow magnetic field interaction in the semi-conducting region of giant planets. Icarus, 296, 59-72. doi: 10 .1016/j.icarus.2017.05.015

Clarke, A. C. (1972). The Wind from the Sun. Harcourt Brace Jovanovich, Inc., New York.

Conrath, B. J., Gierasch, P. J., \& Leroy, S. S. (1990). Temperature and circulation in the stratosphere of the outer planets. Icarus, 83, 255-281. doi: 10.1016/0019 $-1035(90) 90068-\mathrm{K}$

Conrath, B. J., Gierasch, P. J., \& Ustinov, E. A. (1998). Thermal Structure and Para Hydrogen Fraction on the Outer Planets from Voyager IRIS Measurements. Icarus, 135, 501-517. doi: 10.1006/icar.1998.6000

Conrath, B. J., \& Pirraglia, J. A. (1983). Thermal structure of Saturn from Voyager infrared measurements - Implications for atmospheric dynamics. Icarus, 53, 286-292. doi: 10.1016/0019-1035(83)90148-3

de Pater, I., Sault, R. J., Butler, B., DeBoer, D., \& Wong, M. H. (2016, June). Peering through Jupiter's clouds with radio spectral imaging. Science, 352, 11981201. doi: $10.1126 /$ science.aaf2210

de Pater, I., Sault, R. J., Moeckel, C., Moullet, A., Wong, M. H., Goullaud, C., ... Villard, E. (2019, Oct). First ALMA Millimeter-wavelength Maps of Jupiter, with a Multiwavelength Study of Convection. Astronomical Journal, 158(4), 139. doi: 10.3847/1538-3881/ab3643

de Pater, I., Sault, R. J., Wong, M. H., Fletcher, L. N., DeBoer, D., \& Butler, B. (2019, Apr). Jupiter's ammonia distribution derived from VLA maps at 3-37 GHz. Icarus, 322, 168-191. doi: 10.1016/j.icarus.2018.11.024

Dowling, T. E. (1995, October). Estimate of Jupiter's deep zonal-wind profile from Shoemaker-Levy 9 data and Arnol'd's second stability criterion. Icarus, 117, 439-442. doi: 10.1006/icar.1995.1169

Dowling, T. E. (2020, March). Jupiter-style Jet Stability. The Planetary Science Journal, 1(1), 6. doi: 10.3847/PSJ/ab789d

Dowling, T. E., \& Ingersoll, A. P. (1989, November). Jupiter's Great Red SPOT as a shallow water system. Journal of Atmospheric Sciences, 46, 3256-3278. doi: 10.1175/1520-0469(1989)046

Duer, K., Galanti, E., \& Kaspi, Y. (2020, August). The Range of Jupiter's Flow Structures that Fit the Juno Asymmetric Gravity Measurements. Journal of Geophysical Research (Planets), 125(8), e06292. doi: 10.1029/2019JE006292

Flasar, F. M. (1986, March). Global dynamics and thermal structure of Jupiter's atmosphere. Icarus, 65(2-3), 280-303. doi: 10.1016/0019-1035(86)90140-5

Fletcher, L. N., de Pater, I., Reach, W. T., Wong, M., Orton, G. S., Irwin, P. G. J., \& Gehrz, R. D. (2017, April). Jupiter's para- $\mathrm{H}_{2}$ distribution from SOFIA/FORCAST and Voyager/IRIS 17-37 $\mu \mathrm{m}$ spectroscopy. Icarus, 286, 223-240. doi: 10.1016/j.icarus.2016.10.002

Fletcher, L. N., Greathouse, T. K., Orton, G. S., Sinclair, J. A., Giles, R. S., Irwin, P. G. J., \& Encrenaz, T. (2016, November). Mid-infrared mapping of Jupiter's temperatures, aerosol opacity and chemical distributions with IRTF/TEXES. Icarus, 278, 128-161. doi: 10.1016/j.icarus.2016.06.008

Fletcher, L. N., Kaspi, Y., Guillot, T., \& Showman, A. P. (2020). How Well Do We Understand the Belt/Zone Circulation of Giant Planet Atmospheres? Space Science Reviews, 216(2), 30. doi: 10.1007/s11214-019-0631-9

Fletcher, L. N., Orton, G. S., Rogers, J. H., Giles, R. S., Payne, A. V., Irwin, P. G. J., \& Vedovato, M. (2017, April). Moist convection and the 20102011 revival of Jupiter's South Equatorial Belt. Icarus, 286, 94-117. doi: 10.1016/j.icarus.2017.01.001

Fletcher, L. N., Orton, G. S., Teanby, N. A., \& Irwin, P. G. J.

(2009, August). Phosphine on Jupiter and Saturn from Cassini/CIRS. Icarus, 202, 543-564. doi: $10.1016 /$ j.icarus.2009.03.023 
Galanti, E., \& Kaspi, Y. (2021, February). Combined magnetic and gravity measurements probe the deep zonal flows of the gas giants. Monthly Notices of the Royal Astronomical Society, 501(2), 2352-2362. doi: 10.1093/mnras/staa3722

Galanti, E., Kaspi, Y., Duer, K., Fletcher, L., Ingersoll, A. P., Li, C., .. B Bolton, S. J. (2021). Constraints on the latitudinal profile of Jupiter's deep jets. Geophys. Res. Lett., e2021GL092912. doi: 10.1029/2021GL092912

Gierasch, P. J., Ingersoll, A. P., Banfield, D., Ewald, S. P., Helfenstein, P., SimonMiller, A., .. Galileo Imaging Team (2000, February). Observation of moist convection in Jupiter's atmosphere. Nature, 403, 628-630. doi: $10.1038 / 35001017$

Gierasch, P. J., Magalhaes, J. A., \& Conrath, B. J. (1986, September). Zonal mean properties of Jupiter's upper troposphere from Voyager infrared observations. Icarus, 67, 456-483. doi: 10.1016/0019-1035(86)90125-9

Giles, R. S., Fletcher, L. N., \& Irwin, P. G. J. (2017, June). Latitudinal variability in Jupiter's tropospheric disequilibrium species: $\mathrm{GeH}_{4}, \mathrm{AsH}_{3}$ and $\mathrm{PH}_{3}$. Icarus, 289, 254-269. doi: 10.1016/j.icarus.2016.10.023

Grassi, D., Adriani, A., Mura, A., Atreya, S. K., Fletcher, L. N., Lunine, J. I., ... Turrini, D. (2020, April). On the Spatial Distribution of Minor Species in Jupiter's Troposphere as Inferred From Juno JIRAM Data. Journal of Geophysical Research (Planets), 125(4), e06206. doi: 10.1029/2019JE006206

Guillot, T., Li, C., Bolton, S. J., Brown, S. T., Ingersoll, A. P., Janssen, M. A., ... Stevenson, D. J. (2020, August). Storms and the Depletion of Ammonia in Jupiter: II. Explaining the Juno Observations. Journal of Geophysical Research (Planets), 125(8), e06404. doi: 10.1029/2020JE006404

Guillot, T., Miguel, Y., Militzer, B., Hubbard, W. B., Kaspi, Y., Galanti, E., ... Bolton, S. J. (2018, March). A suppression of differential rotation in Jupiter's deep interior. Nature, 555, 227-230. doi: 10.1038/nature25775

Guillot, T., Stevenson, D. J., Atreya, S. K., Bolton, S. J., \& Becker, H. N. （2020, August). Storms and the Depletion of Ammonia in Jupiter: I. Microphysics of "Mushballs". Journal of Geophysical Research (Planets), 125(8), e06403. doi: 10.1029/2020JE006403

Hanley, T. R., Steffes, P. G., \& Karpowicz, B. M. ～(2009, July). A new model of the hydrogen and helium-broadened microwave opacity of ammonia based on extensive laboratory measurements. Icarus, 202, 316-335. doi: $10.1016 /$ j.icarus.2009.02.002

Heimpel, M., Gastine, T., \& Wicht, J. (2016, January). Simulation of deep-seated zonal jets and shallow vortices in gas giant atmospheres. Nature Geoscience, 9(1), 19-23. doi: 10.1038/ngeo2601

Heimpel, M., \& Gómez Pérez, N. (2011, July). On the relationship between zonal jets and dynamo action in giant planets. Geophysical Research Letters, 38(14), L14201. doi: 10.1029/2011GL047562

Hess, S. L., \& Panofsky, H. A. (1951). The atmospheres of the other planets. In T. F. Malone (Ed.), (Vol. Compendium of Meteorology, p. 391-398). American Meteorological Society, Boston.

Hockey, T. (1999). Galileo's planet : observing Jupiter before photography. Bristol, Philadelphia: Institute of Physics Publishing.

Holton, J. (2004). An Introduction to Dynamic Meteorology. Academic press.

Iñurrigarro, P., Hueso, R., Legarreta, J., Sánchez-Lavega, A., Eichstädt, G., Rogers, J. H., .. Gómez-Forrellad, J. M. (2020, January). Observations and numerical modelling of a convective disturbance in a large-scale cyclone in Jupiter's South Temperate Belt. $\quad$ Icarus, 336, $113475 . \quad$ doi: 10.1016/j.icarus.2019.113475

Ingersoll, A., Beebe, R., Mitchell, J., Garneau, G., Yagi, G., \& Muller, J. (1981). Interaction of eddies and mean zonal flow on Jupiter as inferred from Voyager 1 and 2 images. Journal of Geophysical Research, 86, 8733-8743. 
Ingersoll, A. P., Adumitroaie, V., Allison, M. D., Atreya, S., Bellotti, A. A., Bolton, S. J., ... Steffes, P. G. (2017, August). Implications of the ammonia distribution on Jupiter from 1 to 100 bars as measured by the Juno microwave radiometer. Geophys. Res. Lett., 44, 7676-7685. doi: 10.1002/2017GL074277

Ingersoll, A. P., J., G. P., D., B., Vasavada, A. R., \& Galileo Imaging Team. (2000, February). Moist convection as an energy source for the large-scale motions in Jupiter's atmosphere. Nature, 403, 630-632. doi: 10.1038/35001021

Ingersoll, A. P., Kanamori, H., \& Dowling, T. E. (1994, June). Atmospheric gravity waves from the impact of comet Shoemaker-Levy 9 with Jupiter. Geophys. Res. Lett., 21(11), 1083-1086. doi: 10.1029/94GL01057

Janssen, M. A., Oswald, J. E., Brown, S. T., Gulkis, S., Levin, S. M., Bolton, S. J., .. Wang, C. C. (2017, November). MWR: Microwave Radiometer for the Juno Mission to Jupiter. $\quad$ Space Sci. Rev., 213, 139-185. Soi: 10.1007/s11214-017-0349-5

Kaspi, Y., Flierl, G. R., \& Showman, A. P. (2009, August). The deep wind structure of the giant planets: Results from an anelastic general circulation model. Icarus, 202(2), 525-542. doi: 10.1016/j.icarus.2009.03.026

Kaspi, Y., Galanti, E., Hubbard, W. B., Stevenson, D. J., Bolton, S. J., Iess, L., ... Wahl, S. M. (2018, March). Jupiter's atmospheric jet streams extend thousands of kilometres deep. Nature, 555, 223-226. doi: 10.1038/nature25793

Kaspi, Y., Galanti, E., Showman, A. P., Stevenson, D. J., Guillot, T., Iess, L., \& Bolton, S. J. (2020, June). Comparison of the Deep Atmospheric Dynamics of Jupiter and Saturn in Light of the Juno and Cassini Gravity Measurements. Space Sci. Rev., 216(5), 84. doi: 10.1007/s11214-020-00705-7

Li, C., \& Chen, X. (2019, Jan). Simulating Non-hydrostatic atmospheres on Planets (SNAP): formulation, validation and application to the Jovian atmosphere. arXiv e-prints, arXiv:1901.02955.

Li, C., Ingersoll, A., Bolton, S., Levin, S., Janssen, M., Atreya, S., ... Zhang, Z. (2020, February). The water abundance in Jupiter's equatorial zone. Nature Astronomy, 4, 609-616. doi: 10.1038/s41550-020-1009-3

Li, C., Ingersoll, A., Janssen, M., Levin, S., Bolton, S., Adumitroaie, V., ... Williamson, R. (2017, June). The distribution of ammonia on Jupiter from a preliminary inversion of Juno microwave radiometer data. Geophys. Res. Lett., 44, 5317-5325. doi: 10.1002/2017GL073159

Li, C., \& Ingersoll, A. P. (2015, May). Moist convection in hydrogen atmospheres and the frequency of Saturn's giant storms. Nature Geoscience, 8, 398-403. doi: 10.1038/ngeo2405

Li, C., Oyafuso, F. A., Brown, S. T., Atreya, S. K., Orton, G., Ingersoll, A. P., \& Janssen, M. A. (2017, December). How deep is Jupiter's Great Red Spot? AGU Fall Meeting Abstracts.

Li, L., Ingersoll, A. P., Vasavada, A. R., Simon-Miller, A. A., Achterberg, R. K., Ewald, S. P., .. Flasar, F. M. (2006, December). Waves in Jupiter's atmosphere observed by the Cassini ISS and CIRS instruments. Icarus, 185, 416-429. doi: 10.1016/j.icarus.2006.08.005

Li, L., Ingersoll, A. P., Vasavada, A. R., Simon-Miller, A. A., Del Genio, A. D., Ewald, S. P., .. West, R. A. (2006, April). Vertical wind shear on Jupiter from Cassini images. Journal of Geophysical Research (Planets), 111, 4004. doi: 10.1029/2005JE002556

Lian, Y., \& Showman, A. P. (2008, April). Deep jets on gas-giant planets. Icarus, 194, 597-615. doi: 10.1016/j.icarus.2007.10.014

Limaye, S. S. (1986, March). Jupiter - New estimates of the mean zonal flow at the cloud level. Icarus, 65, 335-352. doi: 10.1016/0019-1035(86)90142-9

Little, B., Anger, C. D., Ingersoll, A. P., Vasavada, A. R., Senske, D. A., Breneman, H. H., ... The Galileo SSI Team (1999, December). Galileo Images of Lightning on Jupiter. Icarus, 142, 306-323. doi: 10.1006/icar.1999.6195 
Liu, J., Goldreich, P. M., \& Stevenson, D. J. (2008, August). Constraints on deepseated zonal winds inside Jupiter and Saturn. Icarus, 196, 653-664. doi: 10 .1016/j.icarus.2007.11.036

Liu, J., \& Schneider, T. (2010, November). Mechanisms of Jet Formation on the Giant Planets. Journal of Atmospheric Sciences, 67, 3652-3672. doi: 10.1175/ 2010JAS3492.1

Lunine, J. I., \& Hunten, D. M. (1987, March). Moist convection and the abundance of water in the troposphere of Jupiter. Icarus, 69(3), 566-570. doi: 10.1016/ 0019-1035(87)90025-X

Magalhães, J. A., Seiff, A., \& Young, R. E. (2002, August). The Stratification of Jupiter's Troposphere at the Galileo Probe Entry Site. Icarus, 158, 410-433. doi: 10.1006/icar.2002.6891

Orsolini, Y., \& Leovy, C. B. (1993, Dec). A Model of Large-Scale Instabilities in the Jovian Troposphere. 1. Linear Model. Icarus, 106(2), 392-405. doi: 10.1006/ icar.1993.1180

Ortiz, J. L., Orton, G. S., Friedson, A. J., Stewart, S. T., Fisher, B. M., \& Spencer, J. R. (1998). Evolution and persistence of $5-\mu \mathrm{m}$ hot spots at the Galileo probe entry latitude. J. Geophys. Res., 103, 23051-23069. doi: 10.1029/98JE00696

Oyafuso, F., Levin, S., Orton, G., Brown, S. T., Adumitroaie, V., Janssen, M., ... Bolton, S. (2020, November). Angular Dependence and Spatial Distribution of Jupiter's Centimeter-Wave Thermal Emission From Juno's Microwave Radiometer. Earth and Space Science, 7(11), e01254.

doi: $10.1029 / 2020 \mathrm{EA} 001254$

Pirraglia, J. A. (1989, May). Dissipationless decay of Jovian jets. Icarus, 79, 196207. doi: 10.1016/0019-1035(89)90116-4

Pirraglia, J. A., Conrath, B. J., Allison, M. D., \& Gierasch, P. J. (1981, August). Thermal structure and dynamics of Saturn and Jupiter. Nature, 292(5825), 677-679. doi: 10.1038/292677a0

Porco, C. C., West, R. A., McEwen, A., Del Genio, A. D., Ingersoll, A. P., Thomas, P., .. Vasavada, A. R. (2003, March). Cassini Imaging of Jupiter's Atmosphere, Satellites, and Rings. Science, 299, 1541-1547. doi: 10.1126/science.1079462

Read, P., Gierasch, P., Conrath, B., Simon-Miller, A., Fouchet, T., \& Yamazaki, Y. (2006). Mapping potential-vorticity dynamics on Jupiter. I: Zonal-mean circulation from Cassini and Voyager 1 data. Q. J. R. Meteorol. Soc., 132, 1577-1603.

Salyk, C., Ingersoll, A., Lorre, J., Vasavada, A., \& Del Genio, A. (2006). Interaction between eddies and mean flow in Jupiter's atmosphere: Analysis of Cassini imaging data. Icarus, 185(2), 430-442.

Sánchez-Lavega, A., Orton, G. S., Hueso, R., García-Melendo, E., Pérez-Hoyos, S., Simon-Miller, A., ... Pujic, Z. (2008, January). Depth of a strong jovian jet from a planetary-scale disturbance driven by storms. Nature, 451, 437-440. doi: $10.1038 /$ nature06533

Sánchez-Lavega, A., Rogers, J. H., Orton, G. S., García-Melendo, E., Legarreta, J., Colas, F., ... Wesley, A. (2017, May). A planetary-scale disturbance in the most intense Jovian atmospheric jet from JunoCam and ground-based observations. Geophys. Res. Lett., 44, 4679-4686. doi: 10.1002/2017GL073421

Seiff, A., Kirk, D. B., Knight, T. C. D., Young, R. E., Mihalov, J. D., Young, L. A., ... Atkinson, D. (1998, September). Thermal structure of Jupiter's atmosphere near the edge of a 5 - $\mu \mathrm{m}$ hot spot in the north equatorial belt. $J$. Geophys. Res., 103, 22857-22890. doi: 10.1029/98JE01766

Showman, A. P., \& de Pater, I. (2005, March). Dynamical implications of Jupiter's tropospheric ammonia abundance. Icarus, 174, 192-204. doi: 10.1016/j.icarus .2004 .10 .004

Showman, A. P., \& Dowling, T. E. (2000, September). Nonlinear Simulations of 
Jupiter's 5-Micron Hot Spots. Science, 289, 1737-1740. doi: 10.1126/science .289 .5485 .1737

Showman, A. P., Gierasch, P. J., \& Lian, Y. (2006, June). Deep zonal winds can result from shallow driving in a giant-planet atmosphere. Icarus, 182, 513-526. doi: 10.1016/j.icarus.2006.01.019

Simon-Miller, A. A., Conrath, B. J., Gierasch, P. J., Orton, G. S., Achterberg, R. K., Flasar, F. M., \& Fisher, B. M. (2006, January). Jupiter's atmospheric temperatures: From Voyager IRIS to Cassini CIRS. Icarus, 180, 98-112. doi: 10.1016/j.icarus.2005.07.019

Spiga, A., Guerlet, S., Millour, E., Indurain, M., Meurdesoif, Y., Cabanes, S., ... Fouchet, T. (2020, Jan). Global climate modeling of Saturn's atmosphere. Part II: Multi-annual high-resolution dynamical simulations. Icarus, 335, 113377. doi: $10.1016 /$ j.icarus.2019.07.011

Stone, P. H. (1976). The meteorology of the Jovian atmosphere. In T. Gehrels \& S. Matthews (Eds.), Iau colloq. 30: Jupiter: Studies of the interior, atmosp here, magnetosphere and satellites (p. 586-618).

Sugiyama, K., Nakajima, K., Odaka, M., Kuramoto, K., \& Hayashi, Y.-Y. $\quad$ (2014, February). Numerical simulations of Jupiter's moist convection layer: Structure and dynamics in statistically steady states. Icarus, 229, 71-91. doi: 10.1016/j.icarus.2013.10.016

Sun, Z.-P., Schubert, G., \& Stoker, C. R. (1991, May). Thermal and humidity winds in outer planet atmospheres. $\quad$ Icarus, 91(1), 154-160. doi: 10.1016/0019-1035(91)90134-F

Thomson, S. I., \& McIntyre, M. E. (2016, March). Jupiter's Unearthly Jets: A New Turbulent Model Exhibiting Statistical Steadiness without LargeScale Dissipation*. Journal of Atmospheric Sciences, 73, 1119-1141. doi: 10.1175/JAS-D-14-0370.1

Tollefson, J., Wong, M. H., Pater, I. d., Simon, A. A., Orton, G. S., Rogers, J. H., ... Marcus, P. S. (2017, November). Changes in Jupiter's Zonal Wind Profile preceding and during the Juno mission. Icarus, 296, 163-178. doi: 10.1016/j.icarus.2017.06.007

Vallis, G. K. (2006). Atmospheric and Oceanic Fluid Dynamics. doi: 10.2277/ 0521849691

von Zahn, U., Hunten, D. M., \& Lehmacher, G. (1998). Helium in Jupiter's atmosphere: Results from the Galileo probe helium interferometer experiment. $J$. Geophys. Res., 103(12), 22815-22830. doi: 10.1029/98JE00695

Weidenschilling, S. J., \& Lewis, J. S. (1973). Atmospheric and cloud structures of the jovian planets. Icarus, 20, 465-476.

West, R., Baines, K., Friedson, A., Banfield, D., Ragent, B., \& Taylor, F. (2004). In jupiter. the planet, satellites and magnetosphere. In (p. 79-104).

Wong, M., Mahaffy, P., Atreya, S., Niemann, H., \& Owen, T. $\quad$ (2004). Updated Galileo probe mass spectrometer measurements of carbon, oxygen, nitrogen, and sulfur on Jupiter. Icarus, 171(1), 153-170.

Wong, M. H. (2009, January). Comment on "Transport of nonmethane hydrocarbons to Jupiter's troposphere by descent of smog particles" by Donald M. Hunten [Icarus 194 (2008) 616 622]. Icarus, 199, 231-235. doi: 10.1016/j.icarus.2008.08.017

Wong, M. H., Simon, A. A., Tollefson, J. W., de Pater, I., Barnett, M. N., Hsu, A. I., .. Fletcher, L. N. (2020, April). High-resolution UV/Optical/IR Imaging of Jupiter in 2016-2019. ApJ Supplement, 247(2), 58. doi: $10.3847 / 1538-4365 / \mathrm{ab} 775 \mathrm{f}$

Yamazaki, Y. H., Read, P. L., \& Skeet, D. R. (2005, April). Hadley circulations and Kelvin wave-driven equatorial jets in the atmospheres of Jupiter and Saturn. Planetary and Space Science, 53, 508-525. doi: 10.1016/j.pss.2004.03.009

Young, R. M., Read, P. L., \& Wang, Y. (2018). Simulating Jupiter's weather 
layer. Part I: Jet spin-up in a dry atmosphere. Icarus. Retrieved from http://www.sciencedirect.com/science/article/pii/S0019103518304391 doi: https://doi.org/10.1016/j.icarus.2018.12.005

Zuchowski, L. C., Yamazaki, Y. H., \& Read, P. L. (2009, April). Modeling Jupiter's cloud bands and decks. 1. Jet scale meridional circulations. Icarus, 200, 548562. doi: 10.1016/j.icarus.2008.11.024 